\title{
Cubic-scaling iterative solution of the Bethe-Salpeter equation for finite systems
}

\author{
M. P. Ljungberg,,${ }^{1,2, *}$ P. Koval, ${ }^{2}$ F. Ferrari, ${ }^{2,3}$ D. Foerster, ${ }^{4}$ and D. Sánchez-Portal ${ }^{5,2}$ \\ ${ }^{1}$ Department of Physics, Phillips-University Marburg, Renthof 5, 35032 Marburg, Germany \\ ${ }^{2}$ Donostia International Physics Center, Paseo Manuel de Lardizabal, 4. E-20018 Donostia-San Sebastián, Spain \\ ${ }^{3}$ Dipartimento di Scienza dei Materiali, Università degli Studi di Milano-Bicocca, I-20125 Milano, Italy \\ ${ }^{4}$ LOMA, Université de Bordeaux 1, 351 Cours de la Liberation, 33405 Talence, France \\ ${ }^{5}$ Centro de Física de Materiales CFM-MPC, Centro Mixto CSIC-UPV/EHU, Paseo Manuel de Lardizabal 5, E-20018 San Sebastián, Spain
}

(Received 20 May 2015; published 17 August 2015)

\begin{abstract}
The Bethe-Salpeter equation (BSE) is currently the state of the art in the description of neutral electronic excitations in both solids and large finite systems. It is capable of accurately treating charge-transfer excitations that present difficulties for simpler approaches. We present a local basis set formulation of the BSE for molecules where the optical spectrum is computed with the iterative Haydock recursion scheme, leading to a low computational complexity and memory footprint. Using a variant of the algorithm we can go beyond the Tamm-Dancoff approximation. We rederive the recursion relations for general matrix elements of a resolvent, show how they translate into continued fractions, and study the convergence of the method with the number of recursion coefficients and the role of different terminators. Due to the locality of the basis functions the computational cost of each iteration scales asymptotically as $O\left(N^{3}\right)$ with the number of atoms, while the number of iterations typically is much lower than the size of the underlying electron-hole basis. In practice we see that, even for systems with thousands of orbitals, the runtime will be dominated by the $O\left(N^{2}\right)$ operation of applying the Coulomb kernel in the atomic orbital representation.
\end{abstract}

DOI: 10.1103/PhysRevB.92.075422

PACS number(s): 71.15.Qe, 33.20.-t, 31.15.ag

\section{INTRODUCTION}

$A b$ initio simulations of optical spectra are essential tools in the study of excited state electronic properties of solids, molecules, and nanostructures. For finite systems timedependent density functional theory (TDDFT) [1] based on local or semilocal functionals is widely used. However, TDDFT fails in certain cases, notably for charge transfer excitations [2] which are essential in, e.g., photovoltaic applications. An alternative to TDDFT is Hedin's $G W$ approximation [3] followed by the solution of the Bethe-Salpeter equation (BSE) [4]. Based on many-body perturbation theory [5,6], the $G W / \mathrm{BSE}$ method is a more systematic approach than TDDFT, and it has been shown to give a qualitatively correct description of excitonic effects in solids [4,7] and charge transfer excitations [8,9].

The Bethe-Salpeter equation is a Dyson-like equation for the two-particle Green's function, or equivalently for the four-point polarizability [10]. Within the field of electronic structure theory, developments of the BSE can be traced back to the beginning of the 1960s [6,11,12], with the first ab initio implementations appearing a couple of decades later [13-15]. The $G W / \mathrm{BSE}$ method has been implemented using plane waves and real space grids [10,16-24], linear combination of atomic orbitals (LCAO) [25-29], and within the full-potential linearized augmented plane-wave framework [30]. In practice, the standard way of solving the BSE is by converting it to an effective eigenvalue problem in a particle-hole basis. Since the size of the particle-hole basis scales quadratically with the number of atoms $N$, a straightforward diagonalization of the BSE Hamiltonian will scale like $O\left(N^{6}\right)$. This very steep scaling makes it difficult to treat large scale systems such

${ }^{*}$ Corresponding author: mathias.ljungberg@gmail.com as nanostructures and realistic models of organic photovoltaic devices. For such systems an improved scaling with the number of atoms would be highly beneficial.

Avoiding an explicit diagonalization of the BSE Hamiltonian can be done by using an iterative method to obtain a few low-lying transitions (e.g., the Davidson method [31,32]), or to directly aim for the spectrum, which can be done frequency by frequency using, for example, the generalized minimal residual method $[31,33,34]$ or for the full spectrum with the Haydock recursion scheme [20,35,36]. Another option is to go over to the time domain and solve the equations of motion by time propagation $[37,38]$. These methods only require matrix-vector products to be performed, and assuming that the number of iterations, or time steps, is much smaller than the size of the particle-hole basis, the asymptotic scaling will be $O\left(N^{4}\right)$. However, setting up the BSE Hamiltonian explicitly will still have the cost of $O\left(N^{5}\right)$, and to avoid this, the matrix-vector products need to be performed on the fly, without explicitly constructing the matrix.

Benedict and Shirley made use of the Haydock recursion method to compute optical spectra in the Tamm-Dancoff approximation (TDA) without actually computing the whole BSE Hamiltonian [23]. This was achieved by using, in addition to the particle-hole basis, a real space grid product basis $|\boldsymbol{x}, \boldsymbol{y}\rangle$, in which the screened direct Coulomb interaction is diagonal (the exchange term is sparse in this representation). The scaling of the algorithm was reported to be $O\left(N^{4}\right)$ with the number of atoms; however, a more careful analysis shows that it can be made to scale like $O\left(N^{3}\right)$ by a proper ordering of the loops [39].

This favorable scaling is heavily based on the use of a real-space representation for the particle-hole states. Similar gains can be obtained with the use of LCAO basis sets, where the same asymptotic scaling can be obtained by making use of the sparsity in both direct and exchange Coulomb interaction terms. It should be mentioned that by using 
additional assumptions of locality, which implies screening away Coulomb matrix elements between basis functions that are spatially far from each other, one could even achieve linear scaling [40]; however, the BSE has so far not been treated with these methods. Another linear scaling approach to many-body theory methods has recently been published by Baer and co-workers that make use of stochastic wave functions together with time propagation [41-43].

In the present publication, we will not venture into the realm of linear scaling but rather make use of the more standard iterative methods that, together with locality, lead to cubic scaling with the number of atoms. We present an iterative algorithm to obtain the BSE spectrum for molecules, making use of localized basis sets both for orbitals and products of orbitals. To go beyond the TDA a pseudo-Hermitian version of the Haydock recursion scheme [20] is used. We derive the recursion relations for general matrix elements of a resolvent and show how they translate into continued fractions. Our method has been interfaced to the SIESTA code [44] which is widely used for ground state density functional theory calculations (as an alternative, we can do all-electron calculation using numerical orbitals in an in-house implementation). For the case of the benzene molecule, as a prototypical example, we present a detailed study of the convergence properties of the iterative method, both within the Tamm-Dancoff approximation and for the full BSE. In particular, we study the effect of different termination schemes. Furthermore, for the sake of clarity, we provide a detailed account of the BSE method itself using our notation. Our algorithm scales asymptotically like $O\left(N^{3}\right)$ with the number of atoms and uses $O\left(N^{2}\right)$ memory. We present proof of principle calculations of our implementation, where the runtime is seen to be dominated by the $O\left(N^{2}\right)$ scaling operations for systems up to several thousand orbitals, and discuss some of the bottlenecks and possible improvements of the scheme.

\section{THEORY}

\section{A. Quasiparticles with the $\boldsymbol{G} \boldsymbol{W}$ approximation}

Before the BSE can be set up and solved, the quasiparticle energies must be obtained from a preceding $G W$ calculation [3]. Since the details of our $G W$ implementation have been published elsewhere $[45,46]$, we will here only give a brief summary of the method. The poles of the one-particle Green's function $G$ for an $N$-electron system occur at the ground and excited states of the corresponding $N+1$ and $N-1$ systems, that is, at the electron addition and removal energies. Hedin's $G W$ approximation connects the (irreducible) polarizability $P$, the noninteracting and interacting Green's functions $\left(G^{0}\right.$ and $G$ ), the screened interaction $W$, and the self-energy $\Sigma$ in a set of closed equations

$$
\begin{aligned}
P\left(\boldsymbol{r}, \boldsymbol{r}^{\prime}, \omega\right) & =-\frac{i}{2 \pi} \int G^{0}\left(\boldsymbol{r}, \boldsymbol{r}^{\prime}, \omega+\omega^{\prime}\right) G^{0}\left(\boldsymbol{r}^{\prime}, \boldsymbol{r}, \omega^{\prime}\right) d \omega^{\prime}, \\
W\left(\boldsymbol{r}, \boldsymbol{r}^{\prime}, \omega\right)= & v\left(\boldsymbol{r}, \boldsymbol{r}^{\prime}\right) \\
& +\int v\left(\boldsymbol{r}, \boldsymbol{r}_{2}\right) P\left(\boldsymbol{r}_{2}, \boldsymbol{r}_{3}, \omega\right) W\left(\boldsymbol{r}_{3}, \boldsymbol{r}^{\prime}, \omega\right) d^{3} r_{2} d^{3} r_{3}, \\
\Sigma\left(\boldsymbol{r}, \boldsymbol{r}^{\prime}, \omega\right) & =\frac{i}{2 \pi} \int G^{0}\left(\boldsymbol{r}, \boldsymbol{r}^{\prime}, \omega+\omega^{\prime}\right) W\left(\boldsymbol{r}, \boldsymbol{r}^{\prime}, \omega^{\prime}\right) d \omega^{\prime},
\end{aligned}
$$

$$
\begin{aligned}
G\left(\boldsymbol{r}, \boldsymbol{r}^{\prime}, \omega\right)= & G^{0}\left(\boldsymbol{r}, \boldsymbol{r}^{\prime}, \omega\right) \\
& +\int G^{0}\left(\boldsymbol{r}, \boldsymbol{r}_{2}, \omega\right) \Sigma\left(\boldsymbol{r}_{2}, \boldsymbol{r}_{3}, \omega\right) G\left(\boldsymbol{r}_{3}, \boldsymbol{r}^{\prime}, \omega\right) d^{3} r_{2} d^{3} r_{3} .
\end{aligned}
$$

In our implementation of the $G W$ method the Green's function is expanded in a basis of numerical atomic orbitals (AOs) of finite support $\left\{f_{a}(\boldsymbol{r})\right\}$,

$$
G\left(\boldsymbol{r}, \boldsymbol{r}^{\prime}, \omega\right)=\sum_{a a^{\prime} b b^{\prime}} f_{a}(\boldsymbol{r}) S_{a a^{\prime}}^{-1} G_{a^{\prime} b^{\prime}}(\omega) S_{b^{\prime} b}^{-1} f_{b}^{*}\left(\boldsymbol{r}^{\prime}\right) .
$$

Here and in the following we explicitly write out the overlaps $S_{a b}=\int f_{a}^{*}(\boldsymbol{r}) f_{b}(\boldsymbol{r}) d^{3} r$ when they appear, the matrix quantities $G_{a b}(\omega)$ are always contravariant, and the placement of the indices as subscripts or superscripts is arbitrary. With this representation of the Green's function $G$, we see that the polarizability (1) involves products of AOs $f_{a}(\boldsymbol{r}) f_{b}^{*}(\boldsymbol{r})$. These products are expanded in an (auxiliary) product basis $\left\{F_{\mu}(\boldsymbol{r})\right\}$ of localized numerical functions $[45,46]$

$$
f_{a}(\boldsymbol{r}) f_{b}^{*}(\boldsymbol{r})=\sum_{\mu} V_{\mu}^{a b} F_{\mu}(\boldsymbol{r}),
$$

where the expansion coefficients $V_{\mu}^{a b}$ and the product basis functions $\left\{F_{\mu}(\boldsymbol{r})\right\}$ are determined by numerically expanding the products around a common center and removing redundant functions by a diagonalization based procedure [47]. Only overlapping pairs of orbitals are considered, making the matrix of expansion coefficients sparse when using AOs of local support. The indices $\left\{a a^{\prime} b b^{\prime}\right\}$ will be reserved for atomic orbitals and $\{\mu, \nu\}$ for product functions of atomic orbitals in the following.

Using the product basis, the polarizability $P\left(\boldsymbol{r}, \boldsymbol{r}^{\prime}, \omega\right)$ is represented similarly to the Green's function (5),

$$
P\left(\boldsymbol{r}, \boldsymbol{r}^{\prime}, \omega\right)=\sum_{\mu \mu^{\prime} v v^{\prime}} F_{\mu}(\boldsymbol{r}) S_{\mu \mu^{\prime}}^{-1} P_{\mu^{\prime} v^{\prime}}(\omega) S_{\nu^{\prime} v}^{-1} F_{\nu}^{*}\left(\boldsymbol{r}^{\prime}\right),
$$

where the overlap of the product functions $S_{\mu \nu}=$ $\int F_{\mu}^{*}(\boldsymbol{r}) F_{v}(\boldsymbol{r}) d^{3} r$ appears. Similarly, it can be seen from Eq. (2) that the matrix elements of the bare $v$ and screened $W$ Coulomb interaction must be expanded in the product basis, while the self-energy $\Sigma$ is expanded in the AO basis. For finite systems both the $\left\{f_{a}(\boldsymbol{r})\right\}$ and the product basis $\left\{F_{\mu}(\boldsymbol{r})\right\}$ can be chosen as real.

The frequency-dependent quantities such as $G_{a b}(\omega)$ and $P_{\mu \nu}(\omega)$ are represented on an even-spaced, real-axis, frequency grid via their corresponding spectral functions. An imaginary part of the energy is added in the Green's function $G^{0}(\omega)$ and polarizability $P(\omega)$ that is sufficient to ensure their smoothness on the chosen frequency grid. The convolutions of spectral functions implied by Eqs. (1) and (3) are computed via fast Fourier transforms. Due to the fast convolutions and the locality of the product basis set, the asymptotic scaling of the algorithm is $O\left(N^{3}\right)$ with the number of atoms $N$ [45]. Finally the Dyson equation (4) is directly solved for each frequency to obtain $G_{a b}(\omega)$. The quasiparticle energies are poles in $G_{a b}(\omega)$ and can in certain cases be determined from inspection of the density of states. This does not give the quasiparticle wave function, however. In this paper we adopt the standard way of proceeding and assume that the Kohn-Sham [48] 
(KS) or Hartree-Fock (HF) eigenfunctions that are used to construct the zeroth order Green's function $G^{0}(\omega)$ are good approximations to the quasiparticle states, so that they can be kept fixed and only the quasiparticle energy corrected. We will here only consider the so-called $G_{0} W_{0}$ approximation where a single iteration of the $G W$ equations is performed without self-consistency. We focus on the KS "starting point" in this section. The KS Hamiltonian is

$$
H^{\mathrm{KS}}=T+V^{\mathrm{ext}}(\boldsymbol{r})+V^{\mathrm{H}}(\boldsymbol{r})+V^{\mathrm{xc}}(\boldsymbol{r})
$$

with $T$ the kinetic energy, $V^{\text {ext }}(\boldsymbol{r})$ the external potential, $V^{\mathrm{H}}(\boldsymbol{r})$ the Hartree potential, and $V^{\mathrm{xc}}(\boldsymbol{r})$ the exchange-correlation potential. The KS eigenfunctions are expanded in the AO basis

$$
\psi_{i}(\boldsymbol{r})=\sum_{a} X_{i a} f_{a}(\boldsymbol{r}),
$$

where $X_{i a}=\sum_{a^{\prime}} S_{a a^{\prime}}^{-1}\left\langle a^{\prime} \mid i\right\rangle$ are the eigenvectors of the generalized eigenvalue problem

$$
\sum_{b} H_{a b}^{\mathrm{KS}} X_{i b}=\epsilon_{i}^{K S} \sum_{b} S_{a b} X_{i b} .
$$

If we additionally assume that the inverse of the Green's function $G$ is diagonal in the KS eigenstates $\psi_{i}(\boldsymbol{r})$, the Dyson equation (4) reduces to a set of scalar equations

$$
G_{i i}(\omega)=\frac{1}{\omega-\epsilon_{i}^{\mathrm{KS}}-\left(\Sigma_{i i}(\omega)-V_{i i}^{\mathrm{xc}}\right)},
$$

where we have subtracted the exchange-correlation potential $V_{i i}^{\mathrm{xc}}$ in order to be able to work with the KS eigenvalues. The (assumed real) poles are then found by identifying the zeros of the denominator, either by a graphical solution if the full frequency-dependent quantities are available, or more commonly, by an expansion of $\Sigma_{i i}(\omega)$ around $\epsilon_{i}^{\mathrm{KS}}$, which leads to

$$
\begin{aligned}
\epsilon_{i}^{G W} & =\epsilon_{i}^{\mathrm{KS}}+Z_{i}\left[\operatorname{Re} \Sigma_{i i}\left(\epsilon_{i}^{\mathrm{KS}}\right)-V_{i i}^{\mathrm{xc}}\right], \\
Z_{i} & =\left(1-\left.\frac{\partial \operatorname{Re} \Sigma_{i i}(\omega)}{\partial \omega}\right|_{\omega=\epsilon_{i}^{\mathrm{KS}}}\right)^{-1} .
\end{aligned}
$$

Since we have access to the full frequency dependence of the self-energy we can use the graphical method, which in principle is more accurate and also has the advantage that problems with satellite peaks can be avoided [49]. For comparison purposes we will also make use of the simpler equation (12).

\section{B. Optical spectra with the Bethe-Salpeter equation}

The directionally averaged absorption cross section of a molecule is given by

$$
\sigma(\omega)=\frac{4 \pi \omega}{3 c} \sum_{m} \operatorname{Im} \alpha_{m m}(\omega)
$$

where $\alpha_{m m^{\prime}}(\omega)$ is the dynamical dipole polarizability tensor given by

$$
\alpha_{m m^{\prime}}(\omega)=-\int d^{3} r d^{3} r^{\prime} r_{m} \chi\left(\boldsymbol{r}, \boldsymbol{r}^{\prime}, \omega\right) r_{m^{\prime}}^{\prime} .
$$

The interacting density response function, or reducible polarizability, $\chi\left(\boldsymbol{r}, \boldsymbol{r}^{\prime}, \omega\right)$ is defined in the time domain as a functional derivative of the density with respect to the change of the external potential: $\chi(\mathbf{1}, \mathbf{2}) \equiv \frac{\delta \rho(\mathbf{1})}{\delta U(\mathbf{2})}$. Numbered bold indices, $\boldsymbol{i}=\left\{\mathbf{r}_{i}, \sigma_{i}, t_{i}\right\}$, refer to space, spin, and time coordinates, whereas plain numbered indices contain space and spin, $i=\left\{\mathbf{r}_{i}, \sigma_{i}\right\} . \chi(\mathbf{1}, \mathbf{2})$ is a two-point quantity and it is directly connected to the noninteracting density response $\chi^{0}(\mathbf{1}, \mathbf{2})$ in random-phase approximation or in TDDFT with semilocal functionals [50]. However, when the Hamiltonian becomes nonlocal in space [as in the case of time-dependent HartreeFock (TDHF), TDDFT with hybrid functionals, or Hedin's $G W$ approximation] one must first find the retarded four-point polarizability $L(\mathbf{1}, \mathbf{2}, \mathbf{3}, \mathbf{4})$, and then obtain the two-point one using the relation $\chi(\mathbf{1}, \mathbf{2})=L\left(\mathbf{1}, \mathbf{1}^{+}, \mathbf{2}, \mathbf{2}\right)$ (see Appendix A).

The four-point polarizability $L(\mathbf{1}, \mathbf{2}, \mathbf{3}, \mathbf{4})$ satisfies the BetheSalpeter equation as derived in Appendix A. In the frequency domain the BSE can be written

$$
\begin{aligned}
L(1,2,3,4 \mid \omega)= & L^{0}(1,2,3,4 \mid \omega) \\
& +\int d(5678) L^{0}(1,2,5,6 \mid \omega) \\
& \times K(5,6,7,8) L(7,8,3,4 \mid \omega)
\end{aligned}
$$

with $L^{0}(1,2,3,4 \mid \omega)$ the noninteracting four-point polarizability and

$$
K(1,2,3,4)=v(1,3) \delta(1,2) \delta(3,4)-W(1,2) \delta(1,3) \delta(2,4),
$$

the BSE kernel. Already here the approximation has been made that the screened interaction $W(1,2)$ is independent of the frequency. Introducing an orthonormal two-particle basis $|i j\rangle$ that has the representation $\langle 1,2 \mid i j\rangle=\psi_{i}(1) \psi_{j}^{*}(2)$ in terms of the quasiparticle spin orbitals, we can expand $L$ as

$$
\begin{aligned}
L(1,2,3,4 \mid \omega) & =\sum_{i j, k l}\langle 1,2 \mid i j\rangle L_{i j, k l}(\omega)\langle k l \mid 3,4\rangle \\
& =\sum_{i j, k l} \psi_{i}(1) \psi_{j}^{*}(2) L_{i j, k l}(\omega) \psi_{k}^{*}(3) \psi_{l}(4),
\end{aligned}
$$

with the matrix elements given by

$$
L_{i j, k l}(\omega)=\int d(1234) \psi_{i}^{*}(1) \psi_{j}(2) L(1,2,3,4 \mid \omega) \psi_{k}(3) \psi_{l}^{*}(4) .
$$

$L^{0}$ is expanded similarly. This leads to the matrix equation

$$
L_{i j, k l}(\omega)=L_{i j, k l}^{0}(\omega)+\sum_{i^{\prime} j^{\prime}, k^{\prime} l^{\prime}} L_{i j, i^{\prime} j^{\prime}}^{0}(\omega) K_{i^{\prime} j^{\prime}, k^{\prime} l^{\prime}} L_{k^{\prime} l^{\prime}, k l}(\omega) .
$$

Equation (19) has to be inverted for each frequency which is computationally cumbersome. Fortunately, with certain approximations, it can be reformulated as an effective eigenvalue problem that only has to be solved once. To proceed with this we choose as our one-particle states the quasiparticle states in which the interacting Green's function $G$ is assumed to be diagonal. This leads to $L^{0}$ being diagonal in the two-particle basis

$$
L_{i j, k l}^{0}(\omega)=\frac{\delta_{i k} \delta_{j l}\left(f_{i}-f_{j}\right)}{\omega-\left(\epsilon_{j}-\epsilon_{i}\right)+i \gamma}
$$


where $f_{i}$ denotes the occupation number of spin orbital $\psi_{i}$. We put the expression (20) in equation (19), rearrange terms, and get after some algebra

$$
L_{i j, k l}(\omega)=\left[(\omega+i \gamma) \delta_{i^{\prime} k^{\prime}} \delta_{j^{\prime} l^{\prime}}-H_{i^{\prime} j^{\prime}, k^{\prime} l^{\prime}}^{\mathrm{BSE}}\right]_{i j, k l}^{-1}\left(f_{k}-f_{l}\right),
$$

where we introduced the frequency-independent BSE Hamiltonian

$$
\begin{aligned}
H^{\mathrm{BSE}} & =\sum_{i j, k l}|i j\rangle H_{i j, k l}^{\mathrm{BSE}}\langle k l|, \\
H_{i j, k l}^{\mathrm{BSE}} & =\left(\epsilon_{j}-\epsilon_{i}\right) \delta_{i k} \delta_{j l}+\left(f_{i}-f_{j}\right) K_{i j, k l} .
\end{aligned}
$$

The matrix $H^{\mathrm{BSE}}$ is non-Hermitian. If we solve for its right eigenvectors and eigenvalues

$$
H^{\mathrm{BSE}}|\lambda\rangle=\epsilon_{\lambda}|\lambda\rangle,
$$

and define expansion coefficients of the eigenvectors in terms of the two-particle basis $A_{i j}^{\lambda}=\langle i j \mid \lambda\rangle$, we can obtain a spectral representation of the interacting polarizability as

$$
L_{i j, k l}(\omega)=\sum_{\lambda, \lambda^{\prime}} \frac{A_{i j}^{\lambda} S_{\lambda, \lambda^{\prime}}^{-1} A_{k l}^{\lambda^{\prime} *}\left(f_{k}-f_{l}\right)}{\omega-\epsilon_{\lambda}+i \gamma} .
$$

Here the overlap of the right eigenvectors $S_{\lambda, \lambda^{\prime}}=\sum_{i j} A_{i j}^{\lambda *} A_{i j}^{\lambda^{\prime}}$ appears because the eigenvectors of a non-Hermitian eigenvalue problem are not, in general, orthogonal. Using equations (14) and (17) and a resolution of the identity in the quasiparticle product states, we can rewrite $\alpha_{m m^{\prime}}(\omega)$ in terms of $L$ as

$$
\alpha_{m m^{\prime}}(\omega)=\sum_{i j k l} D_{i j}^{m *} L_{i j, k l}(\omega) D_{k l}^{m^{\prime}},
$$

with the transition dipoles

$$
\begin{aligned}
D_{i j}^{m} & =\left\langle i j \mid D_{m}\right\rangle=\int d(1) \psi_{i}^{*}(1) r_{m} \psi_{j}(1) \\
& =\delta_{x_{i}, x_{j}} \int d^{3} r \psi_{i}^{*}(\boldsymbol{r}) r_{m} \psi_{j}(\boldsymbol{r}) .
\end{aligned}
$$

Here $\psi_{i}(\boldsymbol{r})$ is the spatial part of $\psi_{i}(1)$, and $x(\sigma)$ is the corresponding spin function. Here we denote the dipole operator as a ket, since in general a normal two-point operator $A$ can be expanded as $A=\sum_{i j} A_{i j}|i\rangle\left\langle j\left|\equiv \sum_{i j}\right| i j\right\rangle A_{i j}$. In the preceding analysis spin is explicit in the orbitals. However, $H^{\mathrm{BSE}}$ is not diagonal in a spin orbital basis. If it is diagonalized in the spin indices (see Appendix B), one singlet and three triplet product functions result, with the singlet one being the only one to have a nonvanishing transition dipole moment and so the one visible in the optical response. In the following we will suppress the spin indices and only work with the space quantities. Because of spin symmetry the coupling elements $K$ are modified with the factor $f^{\mathrm{s} / \mathrm{t}}$ being 2 for the singlet and 0 for the triplet

$$
\begin{aligned}
& K_{i j, k l}=f^{\mathrm{s} / \mathrm{t}} H_{i j, k l}^{\mathrm{ex}}+H_{i j, k l}^{\mathrm{dir}}, \\
& H_{i j, k l}^{\mathrm{ex}}=\int d^{3} r d^{3} r^{\prime} \psi_{i}^{*}(\boldsymbol{r}) \psi_{j}(\boldsymbol{r}) v\left(\boldsymbol{r}, \boldsymbol{r}^{\prime}\right) \psi_{k}\left(\boldsymbol{r}^{\prime}\right) \psi_{l}^{*}\left(\boldsymbol{r}^{\prime}\right), \\
& H_{i j, k l}^{\mathrm{dir}}=-\int d^{3} r d^{3} r^{\prime} \psi_{i}^{*}(\boldsymbol{r}) \psi_{k}(\boldsymbol{r}) W\left(\boldsymbol{r}, \boldsymbol{r}^{\prime}\right) \psi_{j}\left(\boldsymbol{r}^{\prime}\right) \psi_{l}^{*}\left(\boldsymbol{r}^{\prime}\right),
\end{aligned}
$$

and the transition dipoles for the singlet get an additional factor of $\sqrt{2}$ (see Appendix B)

$$
D_{i j}^{m, \text { singlet }}=\sqrt{2} \int d^{3} r \psi_{i}^{*}(\boldsymbol{r}) r_{m} \psi_{j}(\boldsymbol{r}),
$$

and the triplet transition dipole is zero. This means that the dynamic dipole polarizability effectively gets an additional factor of 2 for the singlet transition. Since we always consider the singlet for dipole transitions we can drop the "singlet" superscript and let $D_{i j}^{m}$ refer to Eq. (28). An important simplification to the problem is that, due to the occupation factors, only particle-hole and hole-particle product states contribute to the polarizability (see Appendix B) and we can write the eigenfunctions of $H^{\mathrm{BSE}}$ as

$$
|\lambda\rangle=\sum_{v c}|v c\rangle A_{v c}^{\lambda}+\sum_{v c}|c v\rangle A_{c v}^{\lambda}
$$

Here and in the following the indices $\left\{v v^{\prime}\right\}$ will denote occupied (valence), $\left\{c c^{\prime}\right\}$ empty (conduction, unoccupied), and $\{i j k l\}$ general molecular orbitals. Projecting the eigenvalue equation (23) from the left with $\langle v c|$ and $\langle c v|$ we obtain a matrix equation with the following block structure:

$$
\left(\begin{array}{cc}
H_{v c, v^{\prime} c^{\prime}}^{0}+K_{v c, v^{\prime} c^{\prime}} & K_{v c, c^{\prime} v^{\prime}} \\
-K_{c v, v^{\prime} c^{\prime}} & H_{c v, c^{\prime} v^{\prime}}^{0}-K_{c v, c^{\prime} v^{\prime}}
\end{array}\right)\left(\begin{array}{c}
A_{v^{\prime} c^{\prime}}^{\lambda} \\
A_{c^{\prime} v^{\prime}}^{\lambda}
\end{array}\right)=\epsilon_{\lambda}\left(\begin{array}{c}
A_{v c}^{\lambda} \\
A_{c v}^{\lambda}
\end{array}\right),
$$

where $H_{i j, k l}^{0}=\left(\epsilon_{j}-\epsilon_{i}\right) \delta_{i k} \delta_{j l}$. Using the symmetry properties of the BSE kernel $K_{i j, k l}=K_{j i, l k}^{*}=K_{k l, i j}^{*}$ and of the noninteracting Hamiltonian $H_{i j, k l}^{0}=-H_{j i, l k}^{0}$, we can also write

$$
H^{\mathrm{BSE}}=\left(\begin{array}{cc}
H_{v c, v^{\prime} c^{\prime}}^{0}+K_{v c, v^{\prime} c^{\prime}} & K_{v c, c^{\prime} v^{\prime}} \\
-K_{v c, c^{\prime} v^{\prime}}^{0} & -\left(H_{v c, v^{\prime} c^{\prime}}^{0}+K_{v c, v^{\prime} c^{\prime}}\right)^{*}
\end{array}\right) .
$$

The second form (31) is useful because it leads to computational savings when explicitly setting up the matrix. In the Tamm-Dancoff approximation the off-diagonal blocks in the $H^{\mathrm{BSE}}$ (i.e., the couplings between hole-particle and particle-hole states) are set to zero. This leads to two uncoupled Hermitian eigenvalue equations for $A_{v c}^{\lambda}$ and $A_{c v}^{\lambda}$. Due to the symmetries displayed in Eq. (31) we see that the eigenvalues of the two blocks are related as $\epsilon_{\lambda}^{v c}=-\epsilon_{\lambda}^{c v}$, and the eigenvectors as $A_{c v}^{\lambda}=A_{v c}^{\lambda, *}$, where the superscript refers either to the $\{c v\}$ or the $\{v c\}$ sector. Therefore, only one of the equations needs to be solved, for example the one for the $\{v c\}$ sector: $\sum_{v^{\prime} c^{\prime}} H_{v c, v^{\prime} c^{\prime}}^{\text {res }} A_{v^{\prime} c^{\prime}}^{\lambda}=\epsilon_{\lambda} A_{v c}^{\lambda}$. Using the fact that the eigenvectors are orthogonal for a Hermitian problem, the nonzero blocks of the the four-point polarizability are

$$
\begin{gathered}
L_{v c, v^{\prime} c^{\prime}}^{\mathrm{TDA}}(\omega)=\sum_{\lambda} \frac{A_{v c}^{\lambda} A_{v^{\prime} c^{\prime}}^{\lambda *}}{\omega-\epsilon_{\lambda}+i \gamma}, \\
L_{c v, c^{\prime} v^{\prime}}^{\mathrm{TDA}}(\omega)=-\sum_{\lambda} \frac{A_{v c}^{\lambda *} A_{v^{\prime} c^{\prime}}^{\lambda}}{\omega+\epsilon_{\lambda}+i \gamma} .
\end{gathered}
$$

The TDA is a widely used approximation that, in addition to the computational advantages, often provides good agreement with experimental excitation energies for organic molecules [51-53]. At this point it is interesting to note the 
similarities of the BSE, TDDFT, and time-dependent HartreeFock (TDHF). In TDDFT, although for semilocal functionals it is in principle sufficient to look at the response of the density, one can more generally look at the response of the density matrix as was done by Casida [54]. The resulting equations are very similar to the BSE, with the only difference being that the $G W$ eigenvalues are replaced by $\mathrm{KS}$ eigenvalues, and that the direct term is replaced by a TDDFT exchange-correlation kernel. For semilocal exchange-correlation functionals, and real orbitals, the resulting eigenvalue problem can be reduced to a Hermitian problem of half the size-the preferred formulation of TDDFT in quantum chemistry. However, when Hartree-Fock exchange is included (in hybrid functionals, for example) the reduction to the Hermitian form does not simplify things quite as much, since one needs to take the square root of a full matrix which requires an additional diagonalization. The TDHF response equations have the same structure as the BSE ones with Hartree-Fock eigenvalues and an unscreened direct term. The Tamm-Dancoff approximation is also useful in TDDFT and TDHF. For TDHF with TDA one recovers the configuration interaction singles (CIS) equation. The effective BSE eigenvalue problem can in principle be set up also with a frequency-dependent $W$, but then the problem becomes nonlinear. A solution is then obtained when the BSE Hamiltonian with $W\left(\omega=\epsilon_{\lambda}\right)$ has the eigenvalue $\epsilon_{\lambda}$ which means that repeated diagonalizations must be done frequency by frequency to find all solutions. A simpler scheme is to treat the frequency dependence as a diagonal correction to the static solutions and iterate until self-consistency is obtained [55]. In the present publication we will stick to the standard method with a static $W$ (which also makes for an efficient iterative method as will be discussed in the next section).

To set up and diagonalize the BSE Hamiltonian (31) is feasible only for systems with a few thousand particle-hole pairs. For larger matrices an iterative procedure is essential both for memory and runtime requirements. In the following we describe how the the dynamical dipole polarizability tensor (25) can be computed with a Lanczos-type iterative method.

\section{Continued fraction expression for the BSE polarizability}

Using Eqs. (21) and (25) we can rewrite a matrix element of the dynamical dipole polarizability tensor (14) in a form involving the resolvent of the BSE Hamiltonian

$$
\begin{aligned}
\alpha_{m m^{\prime}}(\omega) & =-\sum_{i j k l} D_{i j}^{m *} L_{i j, k l} D_{k l}^{m^{\prime}} \\
& =-\left\langle D_{m}\left|\left(\omega-H^{\mathrm{BSE}}+i \gamma\right)^{-1}\right| D_{m^{\prime}}^{\prime}\right\rangle,
\end{aligned}
$$

where

$$
\begin{aligned}
\left|D_{m}\right\rangle & =\sum_{i j}|i j\rangle\left\langle i j \mid D_{m}\right\rangle, \\
\left|D_{m^{\prime}}^{\prime}\right\rangle & =\sum_{i j}|i j\rangle\left(f_{i}-f_{j}\right)\left\langle i j \mid D_{m^{\prime}}\right\rangle=\sum_{i j}|i j\rangle F_{i j, i j}\left\langle i j \mid D_{m^{\prime}}^{\prime}\right\rangle .
\end{aligned}
$$

In the last equation $\left\langle i j \mid D_{m}\right\rangle$ refers to the singlet transition dipole in Eq. (28), and we denote the occupation difference matrix by

$$
F_{i j, k l}=\left(f_{i}-f_{j}\right) \delta_{i k} \delta_{j l} .
$$

In the Tamm-Dancoff approximation we only consider $\{v c\}$ states which means that the transition dipoles become

$$
\left|D_{m}^{\mathrm{TDA}}\right\rangle=\left|D_{m}^{\prime \mathrm{TDA}}\right\rangle=\sum_{v c}|v c\rangle\left\langle v c \mid D_{m}\right\rangle
$$

(in the following we drop the TDA superscript since it will be clear from the context if the TDA is used or not).

An attractive method of dealing with resolvents is the Haydock recursion scheme [35], where a diagonal matrix element of a resolvent is efficiently computed from Lanczos recursion coefficients by means of continued fractions. Recently it has been shown that also nondiagonal matrix elements of the resolvent can be computed from the same Lanczos coefficients [19,20,36]. Grüning et al. [20] derived continued fraction representations of the nondiagonal resolvent matrix elements using determinant relations. Two-sided Lanczos schemes were used, as well as the novel pseudoHermitian Lanczos method (which we will discuss in the next section). In the method of Rocca et al. $[19,36]$ the tridiagonal matrix obtained by a two-sided Lanczos scheme (or more recently [56], the pseudo-Hermitian scheme) is explicitly diagonalized to obtain the off-diagonal matrix elements. These schemes require a static effective Hamiltonian, in our case a static $W$, in order for the Lanczos coefficients to be frequency independent and usable to obtain the whole spectrum.

Here we present an alternative derivation of the continued fraction relations that only uses the definition of the inverse of an operator and the orthogonality of the Lanczos vectors. The off-diagonal matrix elements come out naturally in this formulation, and it is straightforwardly extendible to block Lanczos, two-sided Lanczos, and pseudo-Hermitian Lanczos schemes. Our derivation also connects to the theory of relaxation functions, also known as the Mori projection technique, first introduced to describe the Laplace transformed correlation function of dynamical systems [57] and later reformulated by Lee [58] in a form more closely related to the one we use here. Our working expressions turn out to be very close to those of Grüning et al. [20].

We want to compute $\left\langle i\left|(\omega-H)^{-1}\right| j\right\rangle$-a general matrix element of the resolvent of the Hermitian operator $H$, with the frequency $\omega$ in general a complex number. Let us define a frequency-dependent solution vector

$$
|\tilde{j}(\omega)\rangle=(\omega-H)^{-1}|\tilde{j}\rangle,
$$

where $|\tilde{j}\rangle=|j\rangle /\|j\|$ is the normalized $|j\rangle$. The matrix element of the resolvent in terms of the solution vector (37) reads

$$
\left\langle i\left|(\omega-H)^{-1}\right| j\right\rangle=\langle i \mid \tilde{j}(\omega)\rangle|| j|| .
$$

Now we generate a set of orthonormal Lanczos vectors $\left\{\left|f_{n}\right\rangle\right\}$ with the starting state $\left|f_{0}\right\rangle=|\tilde{j}\rangle$, using the standard recursion relations [59]

$$
b_{n+1}\left|f_{n+1}\right\rangle=H\left|f_{n}\right\rangle-\left|f_{n}\right\rangle a_{n}-\left|f_{n-1}\right\rangle b_{n},
$$

with the real coefficients $a_{n}=\left\langle f_{n}|H| f_{n}\right\rangle$ and $b_{n}=$ $\left\langle f_{n-1}|H| f_{n}\right\rangle$. Next we expand the solution vector $|\tilde{j}(\omega)\rangle$ in 
the Lanczos basis

$$
|\tilde{j}(\omega)\rangle=\sum_{n}\left|f_{n}\right\rangle c_{n}(\omega),
$$

where the frequency-dependent expansion coefficients $c_{n}(\omega)$ are given by projection onto the basis

$$
c_{n}(\omega)=\left\langle f_{n} \mid \tilde{j}(\omega)\right\rangle \text {. }
$$

The expansion coefficients $c_{n}(\omega)$ contain the information necessary to compute the sought matrix elements of the resolvent. The diagonal matrix element is especially simple (remembering that $\left|f_{0}\right\rangle=|\tilde{j}\rangle$ ),

$$
\left\langle j\left|(\omega-H)^{-1}\right| j\right\rangle=\langle\tilde{j} \mid \tilde{j}(\omega)\rangle\|j\|^{2}=c_{0}(\omega)\|j\|^{2},
$$

that is, only the zeroth coefficient $c_{0}(\omega)$ is needed.

In the original Haydock recursion scheme only diagonal matrix elements were computed. For our purposes we also need the off-diagonal elements, which can be computed using the higher expansion coefficients

$$
\left\langle i\left|(\omega-H)^{-1}\right| \tilde{j}\right\rangle=\langle i \mid \tilde{j}(\omega)\rangle=\sum_{n}\left\langle i \mid f_{n}\right\rangle c_{n}(\omega)
$$

The projections $\left\langle i \mid f_{n}\right\rangle$ of the vectors $\langle i|$ with the Lanczos basis can be computed and saved when the Lanczos vectors are available, thus avoiding the storage of more than the last two vectors. As we shall see, the coefficients $c_{n}(\omega)$ can be computed from continued fractions. An advantage of using continued fractions is that one can terminate them in a physically sensible way which can reduce the number of Lanczos vectors one has to explicitly compute. Projecting the Hermitian transpose of Eq. (39) onto the solution vector $|\tilde{j}(\omega)\rangle$ gives

$$
\begin{aligned}
b_{n+1}\left\langle f_{n+1} \mid \tilde{j}(\omega)\right\rangle= & \left\langle f_{n}|H| \tilde{j}(\omega)\right\rangle-a_{n}\left\langle f_{n} \mid \tilde{j}(\omega)\right\rangle \\
& -b_{n}\left\langle f_{n-1} \mid \tilde{j}(\omega)\right\rangle .
\end{aligned}
$$

Applying the operator $H$ onto the solution vector gives

$$
H|\tilde{j}(\omega)\rangle=\omega|\tilde{j}(\omega)\rangle-|\tilde{j}\rangle .
$$

which follows directly from the definition of the inverse

$$
(\omega-H)(\omega-H)^{-1}=\mathbb{1}
$$

together with the definition of the solution vector $|\tilde{j}(\omega)\rangle(37)$. Inserting Eq. (45) into Eq. (44) we obtain a recursion relation for the expansion coefficients $c_{n}(\omega)$,

$$
b_{n+1} c_{n+1}(\omega)=\omega c_{n}(\omega)-\delta_{n 0}-a_{n} c_{n}(\omega)-b_{n} c_{n-1}(\omega) .
$$

For $n=0$ the relation can be rearranged to give

$$
c_{0}(\omega)=\left[\omega-a_{0}-b_{1} c_{1}(\omega) c_{0}^{-1}(\omega)\right]^{-1},
$$

while for $n>0$ we obtain

$$
c_{n}(\omega) c_{n-1}^{-1}(\omega) b_{n}^{-1}=\left[\omega-a_{n}-b_{n+1} c_{n+1}(\omega) c_{n}^{-1}(\omega)\right] .
$$

We now introduce the relaxation functions of order $n \varphi_{n}(\omega)[57,58]:$

$$
\begin{aligned}
& \varphi_{0}(\omega)=c_{0}(\omega), \\
& \varphi_{n}(\omega)=c_{n}(\omega) c_{n-1}^{-1}(\omega) b_{n}^{-1}, \quad n>0 .
\end{aligned}
$$

After inserting the expansion coefficients (50) in Eqs. (48) and (49) we obtain the continued fraction relations familiar from the Haydock recursion scheme:

$$
\varphi_{n}(\omega)=\left[\omega-a_{n}-b_{n+1}^{2} \varphi_{n+1}(\omega)\right]^{-1} .
$$

After the relaxation functions have been computed for a certain frequency, the expansion coefficients $c_{n}(\omega)$ can be recovered by inverting the relation (50):

$$
\begin{aligned}
c_{n}(\omega) & =\varphi_{n}(\omega) b_{n} c_{n-1}(\omega) \\
& =\varphi_{n}(\omega) b_{n} \varphi_{n-1}(\omega) b_{n-1} \cdots \varphi_{1}(\omega) b_{1} \varphi_{0}(\omega) .
\end{aligned}
$$

In summary, first the coefficients $a_{n}$ and $b_{n}$, as well as the needed projections $\left\langle i \mid f_{n}\right\rangle$ are obtained from Eq. (39), then for each $\omega$ (adding a small positive imaginary part, as appropriate for the retarded response), the relaxation functions $\varphi_{n}(\omega)$ are computed from Eq. (51) using a properly chosen terminator. Then, the expansion coefficients $c_{n}(\omega)$ are obtained from Eq. (52). Finally, the matrix elements are computed from Eqs. (42) and (43).

\section{Iterative BSE without the Tamm-Dancoff approximation}

The full BSE Hamiltonian is non-Hermitian, which means that the Lanczos procedure outlined above must be modified. A two-sided Lanczos procedure where both left and right eigenvectors are generated in the recursive procedure can be used. Ge et al. [56] note that such schemes suffer from instability issues due to the loss of biorthogonality of the current left and right Lanczos vectors, sometimes leading to a breakdown of the procedure. In the Hermitian Lanczos scheme this cannot occur since the right and left Lanczos vectors are the same. However, even in the Hermitian case loss of orthogonality to earlier Lanczos vectors is a known problem that has other unwanted effects such as doubling of the eigenvalues, etc., [60,61]. A way to avoid these problems is to perform an explicit reorthogonalization, which requires the storage of all the Lanczos vectors. The continued fraction representation has been seen to be very stable against these orthogonality problems and we here choose not to do any reorthogonalization. A perhaps more severe disadvantage of the two-sided Lanczos scheme is that it involves twice the number of applications of the Hamiltonian, compared to the Hermitian scheme. Recently, a pseudo-Hermitian algorithm was published that exploits the structure of the BSE eigenproblem to convert it into a Hermitian problem in a special scalar product [20]. In this algorithm one avoids the 
extra multiplication of the Hamiltonian that is present in the two-sided scheme. The problems with loss of biorthogonality are also alleviated [56]. Below we summarize the pseudoHermitian algorithm in our notation.

An operator $A$ is pseudo-Hermitian [62] with respect to the invertible Hermitian operator $\eta$, if

$$
A=\eta^{-1} A^{\dagger} \eta
$$

This means that $\eta A$ is Hermitian, or equivalently that $A$ is Hermitian under the scalar product $\langle\cdot \mid \cdot\rangle_{\eta}=\langle\cdot \mid \eta \cdot\rangle$, provided that the metric $\eta$ is positive definite so that the scalar product is well defined. Furthermore, the eigenvalues of $A$ are real if it is pseudo-Hermitian with respect to an operator that can be written like $\eta=O O^{\dagger}$ with $O$ an invertible operator [63], and such a factorization can always be found for a positive definite $\eta$. If $A$ is a product of two Hermitian operators $A=B C$, then $A$ is pseudo-Hermitian with $B^{-1}$ and $C$, which can be checked using Eq. (53). The BSE Hamiltonian $H^{\mathrm{BSE}}$ given by Eq. (22) can be written in matrix form

$$
H^{\mathrm{BSE}}=H^{0}+F K,
$$

with $F$ given by Eq. (35). Since $F^{2}=I$ we can write

$$
H^{\mathrm{BSE}}=F \bar{H}
$$

where

$$
\bar{H}=F H^{0}+K \text {. }
$$

Since $F H^{0}$ is diagonal and real, and $K_{i j, k l}=K_{k l, i j}^{*}$, it follows that $\bar{H}$ is Hermitian. From the preceding discussion it is clear that $H^{\mathrm{BSE}}$ is pseudo-Hermitian with respect to $\eta=F^{-1}=F$ or $\eta=\bar{H}$. Since $F$ is not positive definite it does not serve as a metric for a scalar product. $\bar{H}$, however, should be positive definite unless there exist singlet-triplet instabilities [51,52,64]. Such instabilities do occur for molecules, and especially for triplet excitations $\bar{H}$ can lose its positive definiteness. This will make the pseudo-Hermitian algorithm fail. However, since in this case also direct diagonalization gives unphysical results one should not view this failure as a drawback of the method.

Within the pseudo-Hermitian Lanczos scheme the same steps are followed as in the Hermitian case. The only difference is that the scalar product is changed from the ordinary $\langle\cdot \mid \cdot\rangle$ to $\langle\cdot \mid \bar{H} \cdot\rangle$, with the Lanczos vectors orthonormal in this product. This means that Eq. (39) stays the same, but the Lanczos coefficients are modified to $a_{n}=\left\langle f_{n}\left|\bar{H} H^{\mathrm{BSE}}\right| f_{n}\right\rangle$ and $b_{n}=$ $\left\langle f_{n-1}\left|\bar{H} H^{\mathrm{BSE}}\right| f_{n}\right\rangle$, which can be seen by multiplying Eq. (39) by $\bar{H}$ and using the orthogonality of the Lanczos vectors in the $\langle\cdot \mid \bar{H} \cdot\rangle$ scalar product. To make the starting vector normalized, it is chosen as $\left|f_{0}\right\rangle=|\tilde{j}\rangle=|j\rangle\langle j|\bar{H}| j\rangle^{-1 / 2}$.

Due to the metric introduced in our scalar product we effectively have right and left Lanczos vectors, related by $\left|f_{n}^{L}\right\rangle=\bar{H}\left|f_{n}^{R}\right\rangle$, and $\left|f_{n}^{R}\right\rangle=\left|f_{n}\right\rangle$, although only one set of vectors is necessary in the actual computation. The resolution of the identity in the Lanczos vectors is

$$
1=\sum_{n}\left|f_{n}^{R}\right\rangle\left\langle f_{n}^{L}\left|=\sum_{n}\right| f_{n}^{R}\right\rangle\left\langle f_{n}^{R}\left|\bar{H}=\sum_{n}\right| f_{n}\right\rangle\left\langle f_{n}\right| \bar{H},
$$

which means that the matrix element of the resolvent must be computed as

$$
\begin{aligned}
\left\langle i\left|(\omega-H)^{-1}\right| \tilde{j}\right\rangle & =\sum_{n}\left\langle i \mid f_{n}\right\rangle\left\langle f_{n}|\bar{H}| \tilde{j}(\omega)\right\rangle \\
& =\sum_{n}\left\langle i \mid f_{n}\right\rangle c_{n}^{\bar{H}}(\omega) .
\end{aligned}
$$

Here $c_{n}^{\bar{H}}(\omega)=\left\langle f_{n}|\bar{H}| \tilde{j}(\omega)\right\rangle$ replaces Eq. (41)-the other equations that are needed can be derived as in the Hermitian case, only replacing the scalar product. Here, even if we only want a diagonal matrix element we have to sum over the projections of all the Lanczos vectors, because the starting (right) vector is not orthogonal (in the ordinary scalar product) to the other Lanczos vectors.

The iterative scheme as it is presented here is general and independent of the basis set used. The efficiency and scaling properties of the method will be determined by the number of operations it takes to apply the effective Hamiltonian to a vector. Unlike other implementations of similar schemes $[19,20,23,36]$, we use local basis sets that will allow us to apply the BSE Hamiltonian to a vector with an asymptotic $O\left(N^{3}\right)$ scaling with the number of atoms. Even though the method does not give eigenvalues and eigenvectors of individual transitions, the excitation energies can be recovered by identifying the peak positions using a very small broadening. For dipole-forbidden transitions, as well as triplet excitations, we can also find the peak positions the same way by computing the density of states, where all transitions are visible. This is achieved by artificially setting all transition dipole matrix elements to unity. The eigenvectors of the transitions are harder to get, and we believe it is more efficient to use, e.g., the Davidson method [31,32] in order to obtain the eigenpairs for a few selected transitions.

\section{Implementation of the Bethe-Salpeter equation}

Having a general description of the BSE and of an iterative algorithm for solving it, we will describe our implementation using local basis functions.

\section{Noniterative algorithm}

It is straightforward to compute the matrix in Eq. (31) and diagonalize it to obtain the four-point polarizability from Eqs. (24) and (25). The matrix elements of the kernel $K$ are computed using Eq. (27). The construction of the matrix requires $O\left(N^{5}\right)$ operations ( $N$ being the number of atoms) and $O\left(N^{4}\right)$ memory for storage. Solving the resulting eigenvalue problem using standard diagonalization techniques gives an even more prohibitive scaling of $O\left(N^{6}\right)$ with the number of atoms. A way to avoid this excessive scaling is to limit the number of electron-hole pairs that are included in the calculation. However, the energy range covered by a constant number of pairs decreases with increasing system size, leading to a deteriorated description of the spectrum. In practice, the limit where explicit diagonalization is feasible is reached for a few tens of atoms: for larger systems iterative schemes are more efficient. Nevertheless, for small systems and for testing purposes straightforward diagonalization is a simple and useful alternative. Using our localized product basis set $\left\{F_{\mu}(r)\right\}$, the 
exchange and direct terms in Eq. (27) take the following form:

$$
\begin{aligned}
H_{i j, k l}^{\mathrm{ex}} & =\sum_{\mu} \tilde{V}_{\mu}^{i j * \sum_{v} \tilde{V}_{v}^{k l} v_{\mu v}}, \\
H_{i j, k l}^{\mathrm{dir}} & =-\sum_{\mu} \tilde{V}_{\mu}^{i k * \sum_{v} \tilde{V}_{v}^{j l} W_{\mu \nu}},
\end{aligned}
$$

where the bare and screened Coulomb matrix elements in the local product basis are

$$
\begin{aligned}
v_{\mu \nu} & =\int d^{3} r d^{3} r^{\prime} F_{\mu}^{*}(\boldsymbol{r}) v\left(\boldsymbol{r}, \boldsymbol{r}^{\prime}\right) F_{\nu}\left(\boldsymbol{r}^{\prime}\right), \\
W_{\mu \nu} & =\int d^{3} r d^{3} r^{\prime} F_{\mu}^{*}(\boldsymbol{r}) W\left(\boldsymbol{r}, \boldsymbol{r}^{\prime}, \omega=0\right) F_{\nu}\left(\boldsymbol{r}^{\prime}\right) .
\end{aligned}
$$

The expansion coefficient $\tilde{V}_{\mu}^{i j}$ of a product of two quasiparticle states is given by

$$
\tilde{V}_{\mu}^{i j}=\sum_{a} X_{i a} \sum_{b} V_{\mu}^{a b} X_{j b}^{*}
$$

where the expansion coefficients $V_{\mu}^{a b}$ are those appearing in Eq. (6). Unlike the local product coefficients $V_{\mu}^{a b}$, the eigenstate product coefficients $\tilde{V}_{\mu}^{i j}$ are not sparse and the equations (59) will scale like $O\left(N^{5}\right)$ if the loops are ordered in the proper way as shown by the boxes [Eq. (61) costs $O\left(N^{4}\right)$ operations]. The singlet transition dipoles can also be calculated from the product functions

$$
D_{i j}^{m}=\sqrt{2} \sum_{\mu} \tilde{V}_{\mu}^{i j} D_{\mu}^{m},
$$

where the dipole moments in the local product basis are $D_{\mu}^{m}=$ $\int d^{3} r F_{\mu}^{*}(\boldsymbol{r}) r_{m}$.

\section{Iterative computation of the BSE}

Let us first look at the TDA which is simpler than the full BSE. Because only the $\{v c\}$ sector needs to be solved, the eigenvalue problem is Hermitian. Moreover, because $\left|D_{m}\right\rangle=$ $\left|D_{m}^{\prime}\right\rangle$ in Eq. (36), we only need to calculate a diagonal matrix element of the resolvent to get the diagonal dynamical dipole polarizability

$$
\begin{aligned}
\alpha_{m m}(\omega) & =-\left\langle\tilde{D}_{m}\left|\left(\omega-H^{\mathrm{BSE}}+i \gamma\right)^{-1}\right| \tilde{D}_{m}\right\rangle \cdot\left\|D_{m}\right\|^{2} \\
& =-c_{0}(\omega+i \gamma) \cdot\left\|D_{m}\right\|^{2} .
\end{aligned}
$$

Here $\left|\tilde{D}_{m}\right\rangle=\left|D_{m}\right\rangle / \| D_{m}||$ in Eq. (34) is used as the starting vector in the Lanczos recursion. The dynamical dipole polarizability can directly be written as a continued fraction using Eqs. (50) and (51):

$$
\alpha_{m m}(\omega)=-\frac{\left\|D_{m}\right\|^{2}}{\omega+i \gamma-a_{0}-\frac{b_{1}^{2}}{\omega+i \gamma-a_{1}-\frac{b_{2}^{2}}{\cdots}} .}
$$

The Lanczos procedure for TDA is

$$
\begin{aligned}
\left|f_{-1}\right\rangle & =0 \\
\left|\tilde{f}_{0}\right\rangle & =\left|\tilde{D}_{m}\right\rangle \\
\left|\tilde{f}_{n+1}\right\rangle & =H^{\mathrm{BSE}}\left|f_{n}\right\rangle-a_{n}\left|f_{n}\right\rangle-b_{n}\left|f_{n-1}\right\rangle \\
b_{n+1} & =\left\langle\tilde{f}_{n+1} \mid \tilde{f}_{n+1}\right\rangle^{1 / 2} \\
\left|f_{n+1}\right\rangle & =\left|\tilde{f}_{n+1}\right\rangle / b_{n+1} \\
a_{n} & =\left\langle f_{n}\left|H^{\mathrm{BSE}}\right| f_{n}\right\rangle
\end{aligned}
$$

where first a non-normalized vector $\left|\tilde{f}_{n+1}\right\rangle$ is computed and the $b_{n+1}^{2}$ coefficient is computed from its norm. The most time-consuming step in computing the Lanczos coefficients is the application of the Hamiltonian to a vector. Generally, we express the Lanczos vector in the $|v c\rangle,|c v\rangle$ basis, similarly to the BSE eigenvectors in Eq. (29),

$$
\left|f_{n}\right\rangle=\sum_{v c}|v c\rangle f_{n}^{v c}+|c v\rangle f_{n}^{c v}
$$

with the expansion coefficients

$$
f_{n}^{v c}=\left\langle v c \mid f_{n}\right\rangle, \quad f_{n}^{c v}=\left\langle c v \mid f_{n}\right\rangle .
$$

In the TDA we only make use of the $|v c\rangle$ functions. We want to find the expansion coefficients of the vector resulting from the application of the Hamiltonian, that is, $\left\langle v c\left|H^{B S E}\right| f_{n}\right\rangle$. The action of $H^{0}$ is evaluated in $O\left(N^{2}\right)$ operations

$$
\left\langle v c\left|H^{0}\right| f_{n}\right\rangle=\left(\epsilon_{c}-\epsilon_{v}\right) f_{n}^{v c} .
$$

To exploit the sparsity in the kernels $H^{\mathrm{ex}}$ and $H^{\text {dir }}$, we also make use of an atomic orbital product basis $|a b\rangle$ with real-space representation $\left\langle\boldsymbol{r} \boldsymbol{r}^{\prime} \mid a b\right\rangle=f_{a}(\boldsymbol{r}) f_{b}^{*}\left(\boldsymbol{r}^{\prime}\right)$. Using the expansion of the quasiparticle states in AOs, Eq. (9), we have

$$
|i j\rangle=\sum_{a b}|a b\rangle X_{i a} X_{j b}^{*},
$$

which allows us to rewrite the kernel as

$$
K_{i j, k l}=\sum_{a b, a^{\prime} b^{\prime}} X_{i a}^{*} X_{j b} K_{a b, a^{\prime} b^{\prime}} X_{k a^{\prime}} X_{l b^{\prime}}^{*},
$$

with the matrix elements of the kernel expressed in the AO basis

$$
\begin{aligned}
& K_{a b, a^{\prime} b^{\prime}}=f^{\mathrm{s} / \mathrm{t}} H_{a b, a^{\prime} b^{\prime}}^{\mathrm{ex}}+H_{a b, a^{\prime} b^{\prime}}^{\mathrm{dir}}, \\
& H_{a b, a^{\prime} b^{\prime}}^{\mathrm{ex}}=\int d^{3} r d^{3} r^{\prime} f_{a}^{*}(\boldsymbol{r}) f_{b}(\boldsymbol{r}) v\left(\boldsymbol{r}, \boldsymbol{r}^{\prime}\right) f_{a^{\prime}}\left(\boldsymbol{r}^{\prime}\right) f_{b^{\prime}}^{*}\left(\boldsymbol{r}^{\prime}\right), \\
& H_{a b, a^{\prime} b^{\prime}}^{\mathrm{dir}}=-\int d^{3} r d^{3} r^{\prime} f_{a}^{*}(\boldsymbol{r}) f_{a^{\prime}}(\boldsymbol{r}) W\left(\boldsymbol{r}, \boldsymbol{r}^{\prime}\right) f_{b}\left(\boldsymbol{r}^{\prime}\right) f_{b^{\prime}}^{*}\left(\boldsymbol{r}^{\prime}\right) .
\end{aligned}
$$

The application of $K$ to a Lanczos vector becomes

$$
\left\langle v c|K| f_{n}\right\rangle=\sum_{a b} X_{v a}^{*} X_{c b} \sum_{a^{\prime} b^{\prime}} K_{a b, a^{\prime} b^{\prime}} \sum_{v^{\prime} c^{\prime}} X_{v^{\prime} a^{\prime}} X_{c^{\prime} b^{\prime}}^{*} f_{n}^{v^{\prime} c^{\prime}} .
$$

The operation is separated in three steps: first the coefficient vector is transformed from the eigenstate basis to the local 
basis

$$
f_{n}^{a b}=\sum_{v} X_{v a} \sum_{c} X_{c b}^{*} f_{n}^{v c}
$$

then the kernel $K$ is applied in the local basis

$$
f_{n}^{\prime a b}=\sum_{a^{\prime} b^{\prime}} K_{a b, a^{\prime} b^{\prime}} f_{n}^{a^{\prime} b^{\prime}}
$$

and finally the coefficient vector is back transformed to the eigenstate basis

$$
\left\langle v c|K| f_{n}\right\rangle=\sum_{a} X_{v a}^{*} \sum_{b} X_{c b} f_{n}^{\prime a b} .
$$

The transform and back-transform can be done in $O\left(N^{3}\right)$ operations since they consist of matrix-matrix multiplications which are done sequentially, as shown by the boxes. The application of the kernel $K_{a b, a^{\prime} b^{\prime}}$ would generally take $O\left(N^{4}\right)$ operations, but due to sparsity it actually takes $O\left(N^{2}\right)$ operations. $H^{\text {ex }}$ is expressed in the $\left\{F_{\mu}(\boldsymbol{r})\right\}$ basis as

$$
H_{a b, a^{\prime} b^{\prime}}^{\mathrm{ex}}=\sum_{\mu, v} V_{\mu}^{a b *} v_{\mu \nu} V_{\nu}^{a^{\prime} b^{\prime}} .
$$

and the action on the coefficients becomes

$$
\sum_{a^{\prime} b^{\prime}} H_{a b, a^{\prime} b^{\prime}}^{\mathrm{ex}} f_{n}^{a^{\prime} b^{\prime}}=\sum_{\mu \ni a, b} \sum_{\nu} \sum_{a^{\prime}, b^{\prime} \in \nu} V_{\mu}^{a b *} v_{\mu \nu} V_{\nu}^{a^{\prime} b^{\prime}} f_{n}^{a^{\prime} b^{\prime}} .
$$

For the direct term we similarly get

$$
H_{a b, a^{\prime} b^{\prime}}^{\mathrm{dir}}=-\sum_{\mu, \nu} V_{\mu}^{a a^{\prime} *} W_{\mu \nu} V_{\nu}^{b b^{\prime}},
$$

and the action on the coefficients is

$$
\sum_{a^{\prime} b^{\prime}} H_{a b, a^{\prime} b^{\prime}}^{\mathrm{dir}} f_{n}^{a^{\prime} b^{\prime}}=\sum_{a^{\prime}, b^{\prime}} \sum_{\mu \ni a, a^{\prime}} \sum_{\nu \ni b, b^{\prime}} V_{\mu}^{a a^{\prime} *} W_{\mu \nu} V_{\nu}^{b b^{\prime}} f_{n}^{a^{\prime} b^{\prime}} .
$$

The Coulomb matrix elements $v_{\mu \nu}$ and $W_{\mu \nu}$ are given by Eq. (60). Because by construction the matrix of product coefficients $V_{\mu}^{a b}$ is sparse, a fixed number of atomic orbitals couple for each $\mu$ or $\nu$ and the operations in Eqs. (77) and (79) scale asymptotically as $O\left(N^{2}\right)$.

For the solution of the full BSE problem we use the pseudoHermitian Lanczos scheme with the scalar product $\langle\cdot \mid \bar{H} \cdot\rangle$ as explained in the previous section. A matrix element of the dynamical dipole polarizability computed with the iterative algorithm is given by

$$
\begin{aligned}
\alpha_{m m^{\prime}}(\omega) & =-\left\langle D_{m}\left|\left(\omega-H^{\mathrm{BSE}}+i \gamma\right)^{-1}\right| D_{m^{\prime}}^{\prime}\right\rangle \\
& =-\sum_{n}\left\langle D_{m} \mid f_{n}\right\rangle c_{n}^{\bar{H}}(\omega+i \gamma)\left\langle D_{m^{\prime}}^{\prime}|\bar{H}| D_{m^{\prime}}^{\prime}\right\rangle^{1 / 2},
\end{aligned}
$$

where the coefficients $c_{n}^{\bar{H}}(\omega)$ are computed from the continued fractions $\varphi_{n}(\omega)$ as given by Eqs. (51) and (52). Note that since we are already computing off-diagonal matrix elements there is little extra cost to obtain the full dynamical dipole polarizability tensor, and not just the diagonal matrix elements as is usually done in the TDA case. The Lanczos procedure in the pseudo-Hermitian case is

$$
\begin{aligned}
\left|f_{-1}\right\rangle & =0, \\
\left|\tilde{f}_{0}\right\rangle & =\left|\tilde{D}_{m^{\prime}}^{\prime}\right\rangle \\
\left|\tilde{f}_{0}^{\prime}\right\rangle & =\bar{H}\left|\tilde{f}_{0}\right\rangle \\
b_{0} & =\left\langle\tilde{f}_{0}^{\prime} \mid \tilde{f}_{0}\right\rangle^{1 / 2}, \\
\left|f_{0}\right\rangle & =\left|\tilde{f}_{0}\right\rangle / b_{0}, \\
\left|f_{0}^{\prime}\right\rangle & =\left|\tilde{f}_{0}^{\prime}\right\rangle / b_{0}, \\
a_{n} & =\left\langle f_{n}^{\prime}|F| f_{n}^{\prime}\right\rangle, \\
\left|\tilde{f}_{n+1}\right\rangle & =F\left|f_{n}^{\prime}\right\rangle-a_{n}\left|f_{n}\right\rangle-b_{n}\left|f_{n-1}\right\rangle, \\
\left|\tilde{f}_{n+1}^{\prime}\right\rangle & =\bar{H}\left|\tilde{f}_{n+1}\right\rangle, \\
b_{n+1} & =\left\langle\tilde{f}_{n+1}^{\prime} \mid \tilde{f}_{n+1}\right\rangle^{1 / 2}, \\
\left|f_{n+1}\right\rangle & =\left|\tilde{f}_{n+1}\right\rangle / b_{n+1}, \\
\left|f_{n+1}^{\prime}\right\rangle & =\left|\tilde{f}_{n+1}^{\prime}\right\rangle / b_{n+1} .
\end{aligned}
$$

In this scheme the intermediate vector $\left|f_{n}^{\prime}\right\rangle$ is saved between iterations in order to minimize the number of applications of the Hamiltonian. To perform a Lanczos iteration we need to apply $\bar{H}$ given by Eq. (56) to some vector $\left|f_{n}\right\rangle$, now containing both particle-hole and hole-particle amplitudes. This in done much in the same way as in the TDA case. The term $F H^{0}$ is diagonal in the eigenstate basis and becomes

$$
\begin{aligned}
& \left\langle v c\left|F H^{0}\right| f_{n}\right\rangle=\sum_{v^{\prime} c^{\prime}}\left(F H^{0}\right)_{v c, v^{\prime} c^{\prime}} f_{n}^{v^{\prime} c^{\prime}}=\left(\epsilon_{v}-\epsilon_{c}\right) f_{n}^{v c}, \\
& \left\langle c v\left|F H^{0}\right| f_{n}\right\rangle=\sum_{c^{\prime} v^{\prime}}\left(F H^{0}\right)_{c v, c^{\prime} v^{\prime}} f_{n}^{c^{\prime} v^{\prime}}=\left(\epsilon_{v}-\epsilon_{c}\right) f_{n}^{c v} .
\end{aligned}
$$

For the coupling matrix elements we transform to the atomic basis as in the TDA case, with the exception that we need to make use of both $|v c\rangle$ and $|c v\rangle$ vectors. The transformation is done for both sets of vectors as shown below:

$$
f_{n}^{a b}=\sum_{c} X_{c a} \sum_{v} X_{v b}^{*}\left(f_{n}^{v c}+f_{n}^{c v}\right)
$$

After the auxiliary vector in Eq. (83) has been computed, the exchange and direct terms are applied in exactly the same way as in the TDA case, and after that the coefficients are back-transformed as

$$
\begin{aligned}
f_{n}^{\prime v c} & =\sum_{a} X_{v a}^{*} \sum_{b} X_{c b} f_{n}^{\prime a b}, \\
f_{n}^{\prime c v} & =\sum_{a} X_{c a}^{*} \sum_{b} X_{v b} f_{n}^{\prime a b} .
\end{aligned}
$$

Finally we need to apply $F$, which is easily done considering its definition (35),

$$
\begin{aligned}
& \left\langle v c|F| f_{n}\right\rangle=\sum_{v^{\prime} c^{\prime}} F_{v c, v^{\prime} c^{\prime}} f_{n}^{v^{\prime} c^{\prime}}=f_{n}^{v c}, \\
& \left\langle c v|F| f_{n}\right\rangle=\sum_{v^{\prime} c^{\prime}} F_{c v, c^{\prime} v^{\prime}} f_{n}^{c^{\prime} v^{\prime}}=-f_{n}^{c v} .
\end{aligned}
$$


To conclude, we have shown that the application of the Hamiltonian onto a particle-hole state takes $O\left(N^{3}\right)$ operations, both when using the Tamm-Dancoff approximation and solving the full BSE. If we use the continued fraction method with a given broadening we can assume that the number of Lanczos coefficients will be independent [23] of the number of atoms. This then leads to an overall $O\left(N^{3}\right)$ complexity scaling of the algorithm.

\section{TEST CALCULATIONS}

\section{A. Simple cases: $\mathbf{N a}_{2}$ and $\mathrm{CH}_{4}$}

As a first test of the implementation we look at two simple test systems for which we can make accurate comparisons to other codes. The sodium dimer is simple in that it has only one valence orbital (filled with two electrons) which makes the spectrum dominated by single transitions. We computed the $G_{0} W_{0} / \mathrm{BSE}$ for this system starting from an all-electron $\mathrm{HF}$ ground state calculation performed with our code, using the cc-pVDZ Gaussian basis set treated as numerical atomic orbitals. The $G_{0} W_{0}$ calculation was performed using a fully frequency-dependent self-energy in the range of the valence and semicore states while the $1 s$ core orbitals were treated with $\mathrm{HF}$ exchange only. The quasiparticle energies were computed using the standard first order expansion of the diagonal $\operatorname{Re} \Sigma_{i i}(\omega)$ around the initial HF eigenvalue $\epsilon_{i}$ according to Eq. (12). This procedure is less accurate than solving for the quasiparticle energy graphically, but here we are more interested in a comparison rather than a fully converged result. The BSE was solved by direct diagonalization. For comparison we use the MOLGW code by Bruneval $[28,65,66]$ where we as far as possible use the same parameters as in our code. In Table I we compare the first ionization potential (IP) and electron affinity (EA) as well as the position of the first BSE transition obtained with the two codes. The agreement is excellent. Furthermore, the computed cross sections for both TDA and full BSE match very well even for higher transitions - the obtained optical spectra lie on top of each other, as can be seen in Fig. 1. Comparing the $G_{0} W_{0}$ IP and EA with the experimental ones (4.92 and $0.43 \mathrm{eV}$, respectively [67]), we get very good agreement for the IP, and reasonable agreement for the EA, considering that the basis set used is far from converged. In total the calculated $G_{0} W_{0}$ quasiparticle gap is overestimated by around $0.2 \mathrm{eV}$, which

TABLE I. Comparison of calculated energies obtained with our code and MOLGW code [28] for the sodium dimer. In both calculations the cc-pVDZ basis set is used. Energies are given in units of eV.

\begin{tabular}{lcr}
\hline \hline & This work & MOLGW \\
\hline IP $(\mathrm{HF})$ & 4.53 & 4.54 \\
EA (HF) & -0.14 & -0.13 \\
Gap $(\mathrm{HF})$ & 4.68 & 4.67 \\
IP $\left(G_{0} W_{0}\right)$ & 4.88 & 4.88 \\
EA $\left(G_{0} W_{0}\right)$ & 0.17 & 0.18 \\
Gap $\left(G_{0} W_{0}\right)$ & 4.71 & 4.70 \\
BSE (TDA), singlet & 2.29 & 2.29 \\
BSE (full), singlet & 2.04 & 2.03 \\
\hline \hline
\end{tabular}

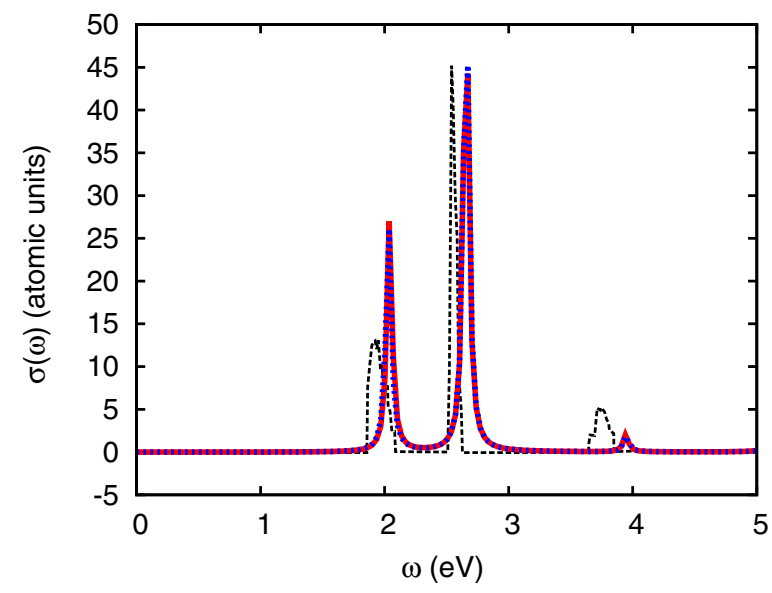

FIG. 1. (Color online) Comparison of the absorption cross section of the sodium dimer between experiment (black dashed line), our method (red full line), and MOLGW (blue dotted line). A Lorentzian broadening with FWHM of $0.05 \mathrm{eV}$ was used in both theoretical spectra. The experimental spectrum (arbitrarily normalized) was taken from Ref. [68], which was adapted from Refs. [69,70].

leads to a too high first optical transition by approximately the same magnitude, as can be seen in Fig. 1.

As a second example we chose to investigate the methane molecule, $\mathrm{CH}_{4}$. Since the first excited states in this system have Rydberg character we augmented the cc-pVDZ basis set with two sets of diffuse $s$ functions (Gaussian exponents of 0.023 and 0.007 ) and one set of $p$ functions (exponent of 0.021 ) centered at the carbon atom [71]. We followed as much as possible the same procedure to extract $G_{0} W_{0}$ eigenvalues between the two codes. In Table II we make the same comparison as in the previous example, with the same excellent agreement for $G W$ energies, first optical excitation in TDA and full BSE, compared to MOLGW. The computed absorption cross sections are also in very good agreement as can be seen in Fig. 2. In order to have an accurate comparison a small Lorentzian broadening with a FWHM of $0.05 \mathrm{eV}$ was used. For this system an experimental comparison is made difficult by large vibrational effects - the lowest-lying states are degenerate and one of them is dissociative [71]. This leads to a smeared out and featureless experimental optical spectrum with an onset at around $9 \mathrm{eV}$ [72]. The experimental

TABLE II. Comparison of calculated energies obtained with our method and the MOLGW code [28] for methane. In both calculations the cc-pVDZ basis set with additional Rydberg functions was used (see text). Energies are given in units of $\mathrm{eV}$.

\begin{tabular}{lcr}
\hline \hline & This work & MOLGW \\
\hline IP (HF) & 14.78 & 14.78 \\
EA (HF) & -0.30 & -0.30 \\
Gap $(H F)$ & 15.08 & 15.08 \\
IP $\left(G_{0} W_{0}\right)$ & 14.41 & 14.41 \\
EA $\left(G_{0} W_{0}\right)$ & -0.28 & -0.28 \\
Gap $\left(G_{0} W_{0}\right)$ & 14.69 & 14.69 \\
BSE (TDA), singlet & 10.85 & 10.85 \\
BSE (full), singlet & 10.84 & 10.84 \\
\hline \hline
\end{tabular}




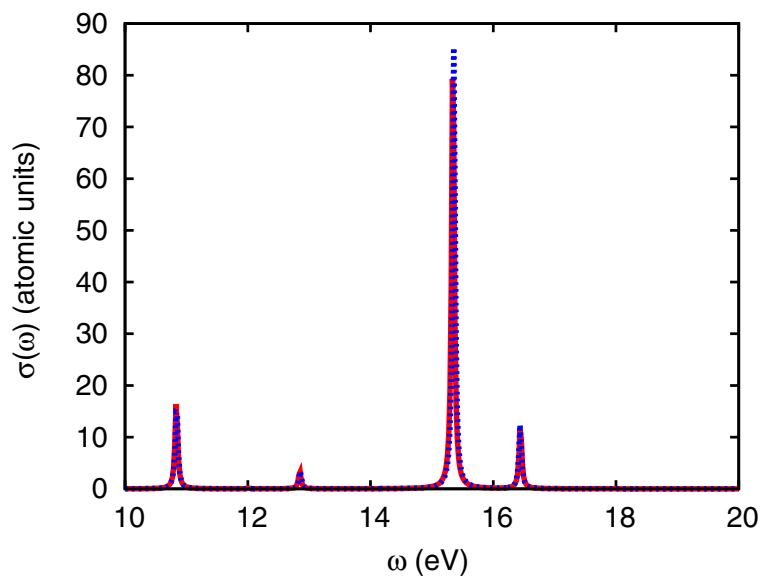

FIG. 2. (Color online) Comparison of the absorption cross section of $\mathrm{CH}_{4}$ between our method (red full line) and MOLGW (blue dotted line). A Lorentzian broadening with FWHM of $0.05 \mathrm{eV}$ was used in both cases.

IP (12.61 eV [67]) is also known to be strongly affected by vibrational effects [73]. Our calculations, corresponding to vertical transitions, thus overestimate the IP and the onset of the spectrum by almost $2 \mathrm{eV}$. However, we are in reasonably good agreement with the theoretical $G W / \mathrm{BSE}$ calculation of Grossman et al. [73] who report an IP of $14.3 \mathrm{eV}$, and the first singlet transition at $10.5 \mathrm{eV}$. In Ref. [71] good agreement with the experimental optical spectrum was achieved using an accurate correlated wave function method as well as the inclusion of vibrational effects that were seen to shift down the onset of the spectrum by around $1.5 \mathrm{eV}$. A shift of similar magnitude is expected for the IP as well. We believe that the inclusion of vibrational effects is absolutely necessary in order to obtain quantitative agreement for this system.

\section{B. Iterative method versus diagonalization}

Confident that our BSE matrix is set up correctly we now turn to the iterative method. As a more suitable test case we chose the benzene molecule that is small enough for direct diagonalization (with a moderately large basis set) while still having many transitions that contribute to the spectrum. The ground state calculation was done with the SIESTA code [44] using the PBE functional and a DZP basis set, using an energy shift parameter of $3 \mathrm{meV}$. Although this basis set is not fully converged for $G W$ quasiparticle energies and optical properties it gives reasonable results for the IP $(8.85 \mathrm{eV})$ and EA $(-1.34 \mathrm{eV})$ compared to earlier obtained results [45], and to experimental values [67]. The first visible optical transition in our calculations occurs at $6.95 \mathrm{eV}$ for the TDA and $6.18 \mathrm{eV}$ for the full BSE, compared to the experimental value of $6.92 \mathrm{eV}$ (extracted from the experiment shown in Ref. [74]). We note that the effect of introducing the TDA here is quite large. A detailed account of the convergence properties of quasiparticle energies and BSE spectra for this system, as well as for larger organic molecules, will appear in a forthcoming publication [49]. For the evaluation of the iterative method, the parameters we choose here are fully sufficient. In Figs. 3 and 4, we show the comparison of the iterative method

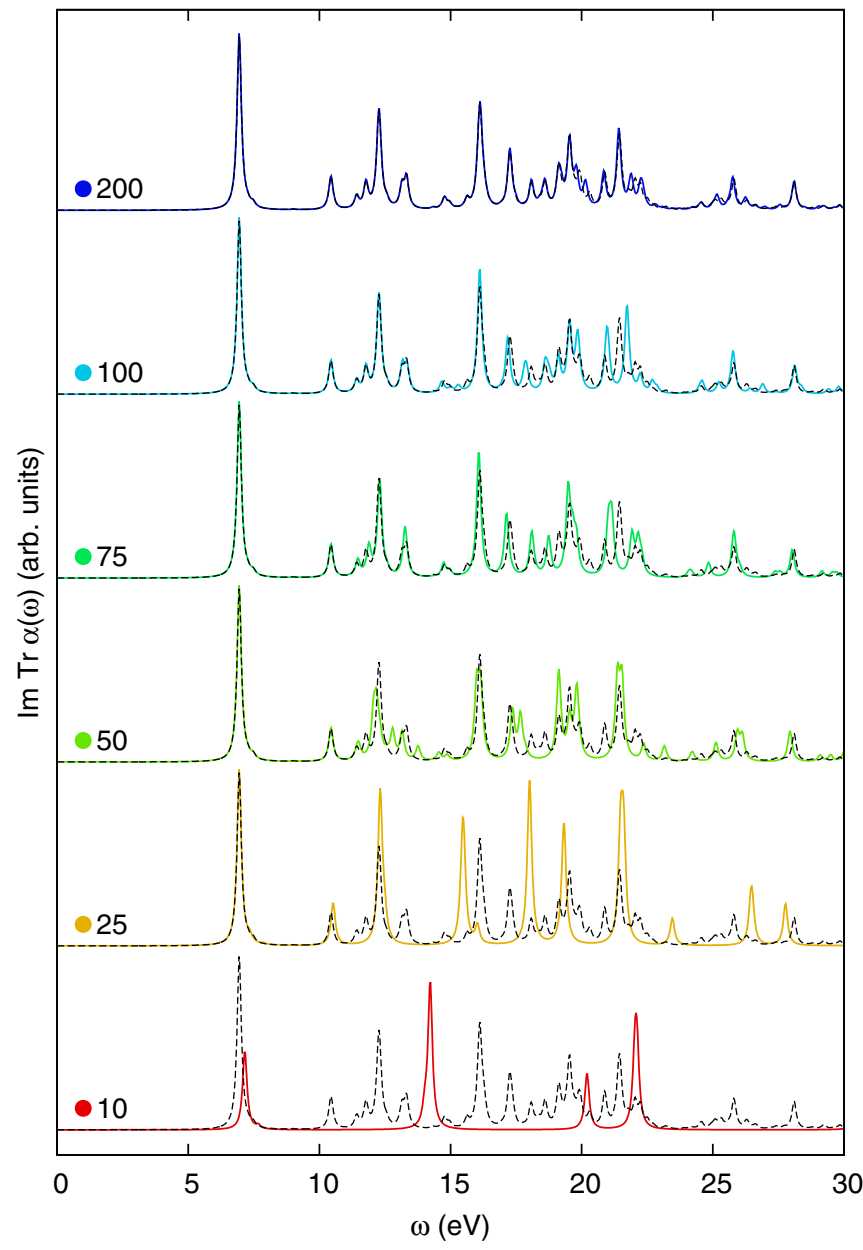

FIG. 3. (Color online) The convergence of the trace of the TDA polarizability with the number of recursion vectors for benzene. The results obtained with $10,25,50,100$, and 200 iterations are compared to direct diagonalization of the full BSE Hamiltonian (dashed lines).

for TDA and full BSE to direct diagonalization. A simple truncation of the continued fraction is used here. We see that the converged iterative spectrum is obtained with around 200 recursion coefficients for TDA and around 400 for the full BSE spectrum for this broadening. Note that the full particle-hole space has a dimension of 1400 for TDA and 2800 for the full BSE.

Next we look at different terminators of the continued fraction. The last relaxation function in Eq. (51) is assumed to satisfy

$$
\varphi_{n-1}(\omega)=\left[\omega-a_{n-1}-b_{n}^{2} \varphi_{T}(\omega)\right]^{-1},
$$

where $\varphi_{T}(\omega)$ is the terminator function. The simplest terminator is obtained by truncation, which means that the remaining coefficients that are not explicitly computed are set to zero. This gives $\varphi_{T}(\omega)=1 / \omega$, and corresponds to a representation of the dynamical dipole polarizability as a sum of $\delta$ functions. However, often a more suitable terminator can be found by extrapolating the remaining coefficients according to some physical model suited to the system of study. In the first model we consider, the dynamical dipole polarizability is assumed to be a continuous distribution without gap, centered at $a$ 


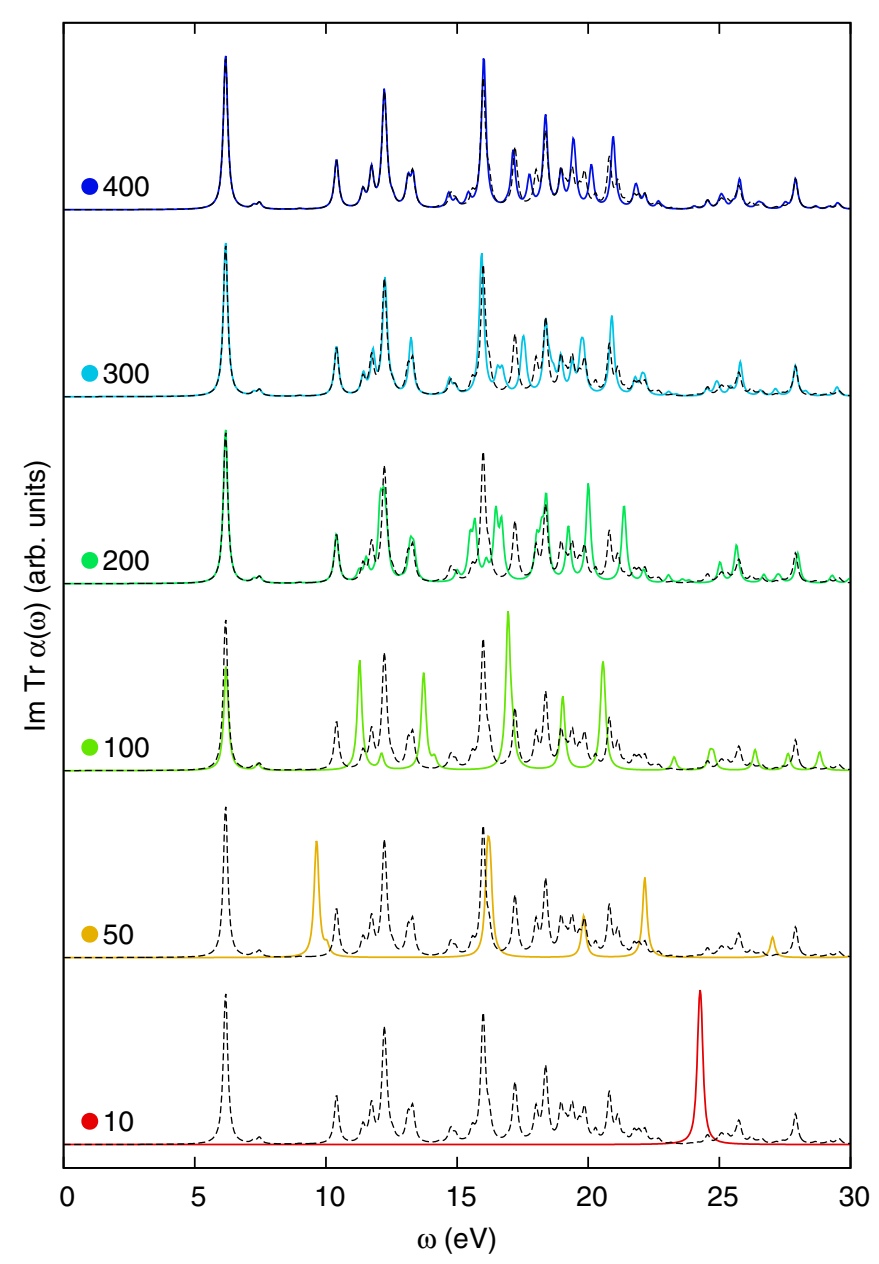

FIG. 4. (Color online) The convergence of the trace of the full BSE polarizability with the number of recursion vectors for benzene. The results obtained with 10, 50, 100, 200, 300, and 400 iterations are compared to direct diagonalization of the BSE Hamiltonian.

and with width $2 E_{W}$. In this case the $a_{n}$ coefficients should converge to $a$, and $b_{n}$ should converge to $b=E_{W} / 2$ [35,75]. At convergence, we get the so-called "self-consistent" (SC) terminator [35]

$$
\varphi_{T}(\omega)=\left[\omega-a-b^{2} \varphi_{T}(\omega)\right]^{-1},
$$

which has the solution

$$
\varphi_{T}(\omega)=\frac{\omega-a-\sqrt{(\omega-a)^{2}-4 b^{2}}}{2 b^{2}},
$$

where the negative root was chosen. In the TDA case we only look at positive energies, so the dynamical dipole polarizability could be approximated (with sufficient broadening) to be a continuous distribution where the terminator (88) can be used. For the full BSE case, however, both positive and negative frequencies are explicitly treated. Since the time-ordered polarizability (as well as the case without any imaginary convergence factor) is symmetric around $\omega=0$ it has at least two distributions separated by a gap.

The presence of the gap in the middle of the distribution is included in the second model we look at. Turchi et al. analyzed the behavior of the recursion coefficients for densities of states with a gap and showed that if $2 E_{G}$ is the gap ( $a$ and $E_{W}$ defined as before) the $a_{n}$ coefficients oscillate with limits $a_{ \pm}=a \pm E_{G}$, and $b_{n}$ with limits $\left(b_{ \pm}=E_{W} \pm E_{G}\right) / 2$ [75]. The period of the oscillations depends on the details of the density of states. If no gap is present, we have the situation of Eq. (88). For a symmetric distribution around the middle of a single gap, which could be a good approximation to the full BSE case, the period is two, and the terminator is

$$
\varphi_{T}(\omega)=\left\{\omega-a_{ \pm}-b_{ \pm}^{2}\left[\omega-a_{\mp}-b_{\mp}^{2} \varphi_{T}(\omega)\right]^{-1}\right\}^{-1},
$$

which has the solution (for the negative root)

$$
\begin{aligned}
\varphi_{T}(\omega) & =-p(\omega) / 2-\sqrt{p^{2}(\omega) / 4-q(\omega)}, \\
p(\omega) & =-\frac{\left(\omega-a_{ \pm}\right)\left(\omega-a_{\mp}-b_{ \pm}^{2}+b_{\mp}^{2}\right)}{\left(\omega-a_{ \pm}\right) b_{\mp}^{2}}, \\
q(\omega) & =\frac{\omega-a_{\mp}}{\left(\omega-a_{ \pm}\right) b_{\mp}^{2}} .
\end{aligned}
$$

We denote this model SC2. Because of symmetry around frequency $\omega=0$ the $a_{n}$ coefficients will oscillate around zero in the full BSE case. Indeed, since only the odd moments of the line shape contribute to $a_{n}$, they should be zero [35,57]. However, in practice, orthogonality between the Lanczos vectors will eventually be lost due to numerical errors, and this introduces nonzero values of $a_{n}$. In practice one can at any point in the recursion sequence make the assumption that the coefficients have converged and so put in the value of the last computed coefficients in Eq. (88) or (90). Another option is to make the assumption that the coefficients will converge to the average value of the already computed coefficients, removing some of the bias of the exact point in the chain the termination was made. We denote the averaged terminators by SC-av and SC2-av when the average is applied for the terminator in (88) or in (90), respectively. When $a_{n}$ is set to zero in Eqs. (88) and (90), the terminator reduces to the one used in Refs. [20,36] (except for the signs of $b_{ \pm}^{2}$ and $b_{\mp}^{2}$ ) which is appropriate for the full BSE case. The consequence of the choice of terminator is illustrated in Figs. 5 and 6 for the TDA and full BSE case, respectively. The dynamical dipole polarizability was computed with 20 and 100 iterations for TDA and full BSE, respectively, while using simple truncation, or terminators defined by Eq. (88) or (90), with or without averaging.

For TDA the self-consistent terminator SC gives a slight improvement while SC2 does better, although it introduces more broadening. When averaging the coefficients we introduce even more broadening in the continuum part of the dynamical dipole polarizability. Looking at the $a_{n}$ and $b_{n}$ coefficients we see that they do not converge but oscillate, which is expected because our small basis set cannot give rise to a continuous dynamical dipole polarizability in the continuum. For an arbitrary sticklike distribution the behavior of the coefficients is complicated. If we look at the averages of the coefficients, we see that $\left\langle a_{n}\right\rangle \approx 72 \mathrm{eV}$, which is close to the center of the spectrum, $65 \mathrm{eV}$, as estimated as half the range of the $G W$ eigenvalues, while $\left\langle b_{n}\right\rangle \approx 33 \mathrm{eV}$, which is close to a quarter of the range of the spectrum, as expected. Averages of the even and odd $b_{n}$ coefficients do not differ almost at all, hence the very similar appearance of the averaged versions of 


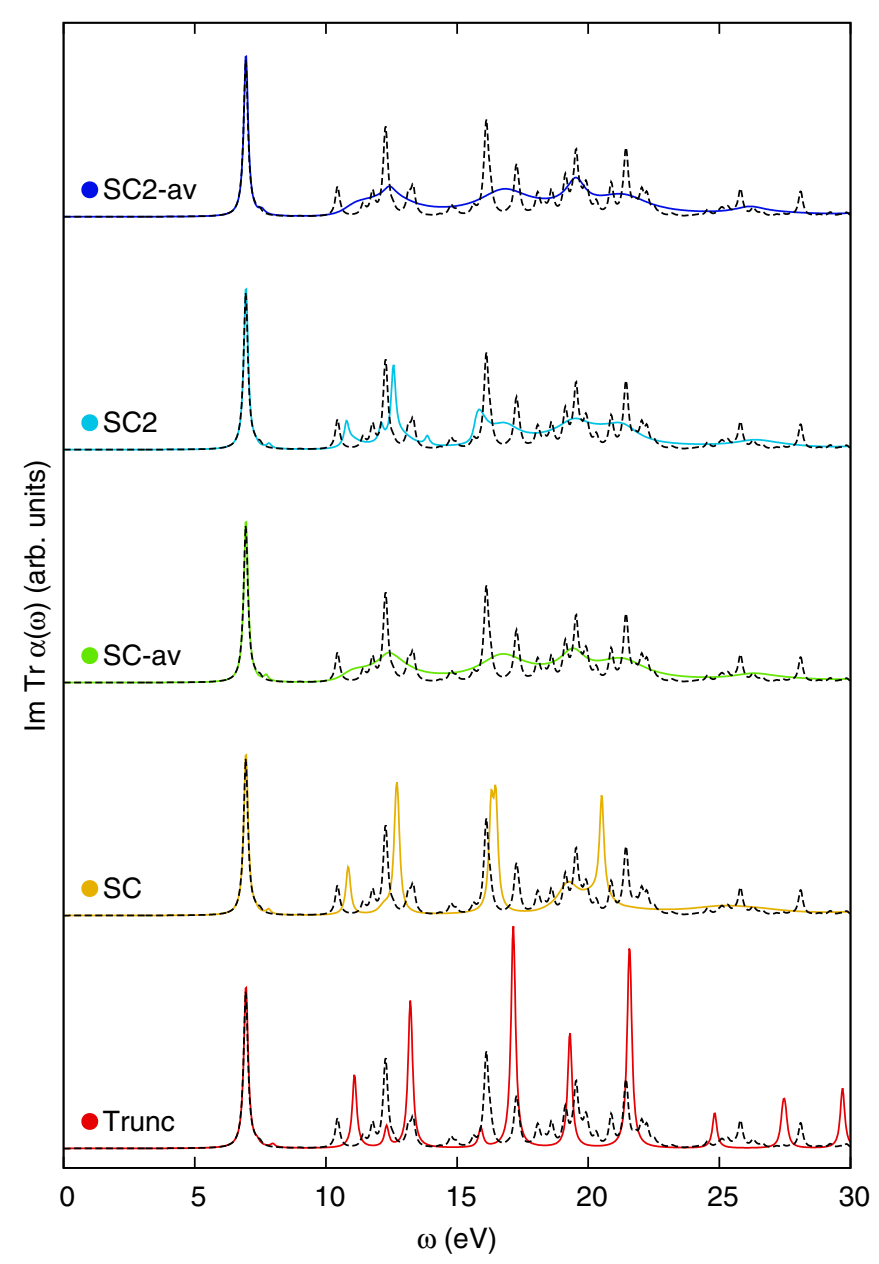

FIG. 5. (Color online) Comparison of different terminators of the continued fraction for the iteratively computed TDA dynamical dipole polarizability of benzene. The number of iterations was set to 20 . See the text for description of the different terminators.

terminators $\mathrm{SC}$ and $\mathrm{SC} 2$. Using two following $b_{n}$ coefficients, however, preserves some oscillations and gives a slightly better agreement to the converged spectrum.

In the full BSE case, the SC terminator fails completely and gives negative intensities. Here it is clear that at least two oscillating coefficients must be used for a reasonable description. $\left\langle a_{n}\right\rangle$ was confirmed to be zero, and the averages of the odd and even coefficients were seen to be 72 and $64 \mathrm{eV}$, respectively. Their difference $(8 \mathrm{eV})$ should correspond to half the gap $E_{G}$, roughly $6 \mathrm{eV}$ in our calculations, estimated from the $G W$ eigenvalues. Here again, we observe that taking the average leads to a smoother spectrum that does not necessarily improve things from only using the last two coefficients. This is likely due to the complicated oscillations coming from the sticklike distribution obtained with our small basis set.

\section{Demonstration of the low scaling with system size}

To demonstrate the scaling properties of our algorithm we performed Lanczos iterations for alkane chains of increasing length. One-dimensional systems are the most favorable cases for algorithms that make use of sparsity, since the number of overlapping functions will be small. To demonstrate the

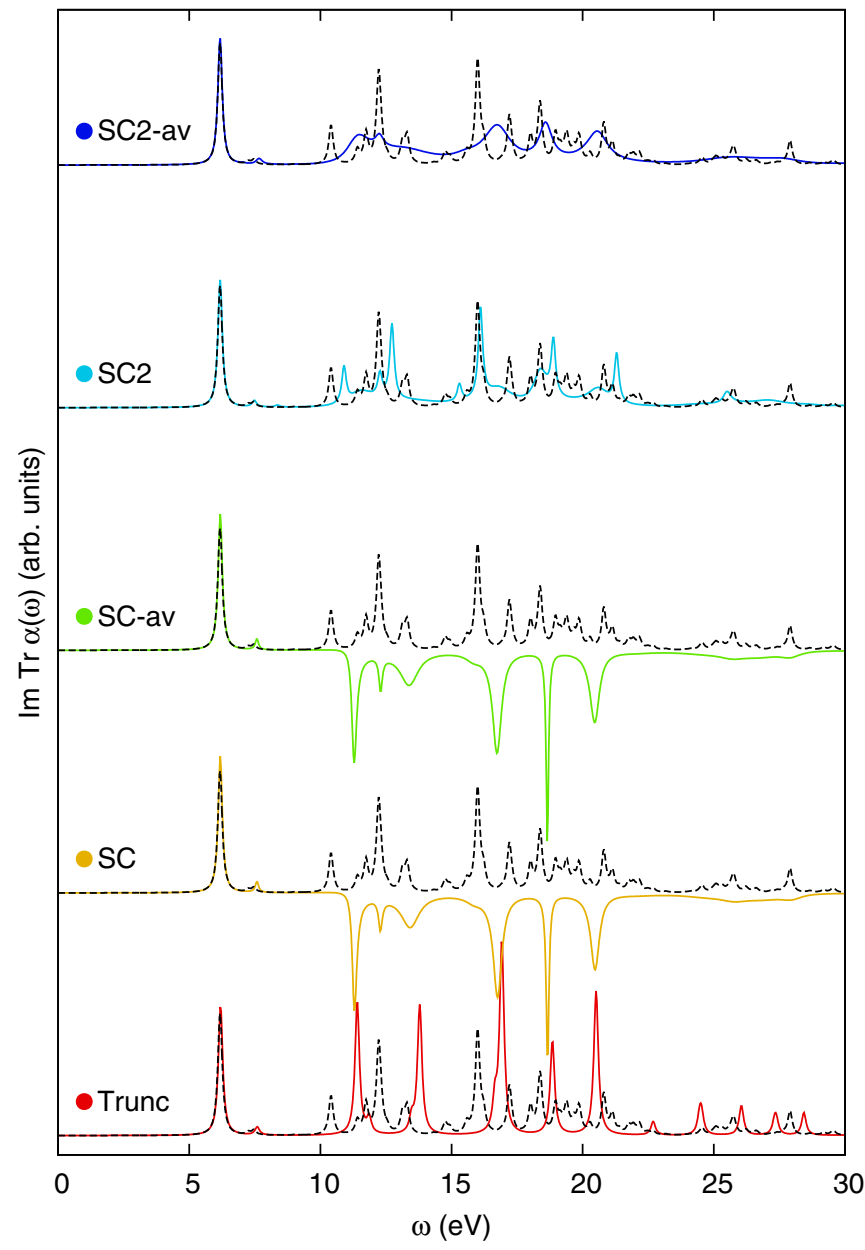

FIG. 6. (Color online) Comparison of different terminators of the continued fraction for the iteratively computed full BSE dynamical dipole polarizability of benzene. The number of iterations was set to 100. See the text for description of the different terminators.

asymptotic scaling of our algorithm this system is also idealthe part that scales cubically depends on the number of AOs, while the dominant quadratic scaling operations involve the number of overlapping AOs. A sparse one-dimensional system maximizes the ratio of the former to the latter. The ground state calculation was done with SIESTA using the LDA functional and a minimal SZ basis set. Although scaling like $O\left(N^{3}\right)$, our $G W$ scheme turned out to be a bottleneck as the systems grow larger, and we therefore chose to bypass the $G W$ step and directly do a TDHF benchmark starting from LDA eigenstates. For the purpose of testing the iterative BSE algorithm the choice of starting point makes no difference. In Fig. 7 we show the runtime, per Lanczos step, or alkane chains of different sizes divided by the runtime of the smallest chain, $C_{64} H_{130}$. The largest alkane chain we considered was $C_{1024} H_{2050}$ with 6146 basis functions. The pseudo-Hermitian algorithm was used in this comparison. In the figure the part of the runtime coming from the basis transformation in Eqs. (83) and (84) that should scale cubically is contrasted to the remaining runtime contributions. For small systems the basis transform is negligible in comparison to the other terms but due to its cubic asymptotic scaling it will eventually start to dominate. We see 


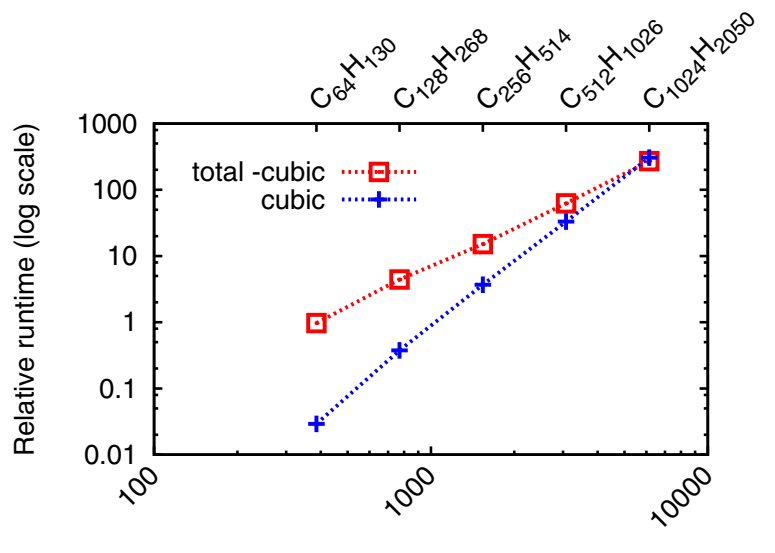

Number of basis functions (log scale)

FIG. 7. (Color online) Runtime per Lanczos vector for alkane chains of different length, divided by the runtime of the $\mathrm{C}_{64} \mathrm{H}_{130}$ chain. The red points are the total runtime minus the runtime of the basis transformation, and the blue the contribution of the basis transformation (denoted cubic). Dotted lines have been drawn between the points as a guide for the eye.

that for the largest chain considered the basis transformation consumes around half the runtime, and we would need to go to even larger systems for the cubic terms to dominate completely. We must here stress the fact that we have used an almost artificially sparse system in order to demonstrate the cubic scaling of the algorithm. For more realistic systems that are less sparse and have more basis functions per atom, the onset where the cubic terms start to dominate will occur much later. We can thus expect that the quadratic and lower terms will dominate for systems with up to several thousands of basis functions, i.e., for most systems that can be practically treated with standard DFT methods.

\section{CONCLUSIONS}

We have described, and implemented, an iterative scheme to compute the optical response of molecular systems at the Bethe-Salpeter level, using local basis sets. We go beyond the Tamm-Dancoff approximation by an extension of the Hermitian Haydock recursion scheme to the pseudo-Hermitian case and provide a derivation of this extension. We show that it is possible to develop an implementation with low scaling with the system size by exploiting the localization of the basis set of numerical atomic orbitals. Proof of principle calculations are shown, focusing on the case of benzene, and the influence of the number of recursion vectors is discussed, as is the effect of different terminators of the continued fractions on the obtained dynamical dipole polarizability.

The theoretical scaling of our method is $O\left(N^{3}\right)$. However, calculations performed for alkane chains containing up to 1024 carbon atoms shows that the contribution of the cubic terms is small. Even for the largest systems considered the contribution of the cubic terms is of comparable magnitude to to that of the quadratic terms coming from the application of the Coulomb kernel in the atomic basis.

What we have presented here is a proof of principles of the method, plus an analysis of the convergence of our iterative
BSE scheme. Our final goal, however, is to create a method (implemented in a suite of programs) capable of accurately investigating complex systems containing thousands of atoms. In order to reach this goal we are currently investigating ways to improve the performance of the method. These include an efficient parallelization scheme and a more optimized basis set for the expansion of atomic orbital products. It is also the case that the $G W$ calculation needed to obtain the quasiparticle energies and states, as well as the screened interaction matrix elements, can benefit from similar improvements.

\section{ACKNOWLEDGMENTS}

We acknowledge support from the Deutsche Forschungsgemeinschaft (DFG) through the SFB 1083 project, the ANR ORGAVOLT project, and the Spanish MINECO MAT201346593-C6-2-P project. Discussions with Mark Casida are gratefully acknowledged. D.F. thanks Lorin X. Benedict for discussions concerning the scaling of the algorithm. P.K. acknowledges financial support from the Fellows Gipuzkoa program of the Gipuzkoako Foru Aldundia through the FEDER funding scheme of the European Union, Una manera de hacer Europa. F.F. acknowledges support from the EXTRA programme of the "Università degli Studi di Milano-Bicocca" and from the Erasmus Placement programme for student mobility.

\section{APPENDIX A: DERIVATION OF THE BETHE-SALPETER EQUATION}

Here we derive the BSE equation following an approach similar to that given in Refs. [76,77]. The purpose of this appendix is to derive the equations using our notation to avoid possible confusions with different notations and conventions that can be found in the literature.

The reducible two-point polarizability is the response of the density to a local perturbation $U$,

$$
\chi(\mathbf{1}, \mathbf{2})=\frac{\delta \rho(\mathbf{1})}{\delta U(\mathbf{2})}=-i \frac{\delta G\left(\mathbf{1}, \mathbf{1}^{+}\right)}{\delta U(\mathbf{2})},
$$

where the "+" superscript denotes the addition of a positive infinitesimal to the time argument. A generalization can be made to the nonlocal response of the interacting Green's function $G$ to a nonlocal perturbation, giving the four-point polarizability

$$
L(\mathbf{1}, \mathbf{2}, \mathbf{3}, \mathbf{4})=-i \frac{\delta G(\mathbf{1}, \mathbf{2})}{\delta U(\mathbf{3}, \mathbf{4})} .
$$

Comparing Eq. (A1) with (A2), we conclude that $\chi(\mathbf{1}, \mathbf{2})=$ $L\left(\mathbf{1}, \mathbf{1}^{+}, \mathbf{2}, \mathbf{2}\right)$. Using the Schwinger functional derivative method (see Refs. [3,78], and references therein) the following relation can be proved:

$$
L(\mathbf{1}, \mathbf{2}, \mathbf{3}, \mathbf{4})=i G(\mathbf{1}, \mathbf{4}, \mathbf{2}, \mathbf{3})-i G(\mathbf{1}, \mathbf{2}) G(\mathbf{4}, \mathbf{3}),
$$

where the two-particle Green's function is defined as

$$
G(\mathbf{1}, \mathbf{2}, \mathbf{3}, \mathbf{4})=(-i)^{2}\left\langle N\left|\mathcal{T}\left\{\hat{\psi}(\mathbf{1}) \hat{\psi}(\mathbf{2}) \hat{\psi}^{\dagger}(\mathbf{4}) \hat{\psi}^{\dagger}(\mathbf{3})\right\}\right| N\right\rangle
$$

Instead of working with the two-particle Green's function we will directly derive the Bethe-Salpeter equation for the 
four-point polarizability $L$. We will use two relations: the chain rule

$$
\frac{\delta F[G[H]](\mathbf{1}, \mathbf{2})}{\delta H(\mathbf{3}, \mathbf{4})}=\int d(\mathbf{5 6}) \frac{\delta F[G](\mathbf{1}, \mathbf{2})}{\delta G(\mathbf{5 , 6})} \frac{\delta G[H](\mathbf{5}, \mathbf{6})}{\delta H(\mathbf{3}, \mathbf{4})},
$$

and a transformation of a derivative of a function to include its inverse

$$
\frac{\delta F(\mathbf{1}, \mathbf{2})}{\delta G(\mathbf{3}, \mathbf{4})}=-\int d(\mathbf{5 6}) F(\mathbf{1}, \mathbf{5}) \frac{\delta F^{-1}(\mathbf{5}, \mathbf{6})}{\delta G(\mathbf{3}, \mathbf{4})} F(\mathbf{6}, \mathbf{2}) .
$$

Using Eq. (A6) we can write

$$
\frac{\delta G(\mathbf{1}, \mathbf{2})}{\delta U(\mathbf{3}, \mathbf{4})}=-\int d(\mathbf{5 6}) G(\mathbf{1}, \mathbf{5}) G(\mathbf{6}, \mathbf{2}) \frac{\delta G^{-1}(\mathbf{5}, \mathbf{6})}{\delta U(\mathbf{3}, \mathbf{4})} .
$$

From the Dyson equation for interacting Green's function $G$ we have

$$
G^{-1}(\mathbf{5}, \mathbf{6})=G_{0}^{-1}(\mathbf{5}, \mathbf{6})-U(\mathbf{5}, \mathbf{6})-v_{\mathrm{H}}(\mathbf{5}) \delta(\mathbf{5}, \mathbf{6})-\Sigma(\mathbf{5}, \mathbf{6}),
$$

where we added the external potential $U$ to the Hamiltonian (it will be set to zero after the derivatives have been taken) and the Hartree potential $v_{\mathrm{H}}$ is taken outside of the noninteracting Green's function $G_{0}$. Evaluating the functional derivative, remembering that $G_{0}$ is independent of $U$, we get

$$
\begin{aligned}
& \frac{\delta G^{-1}(\mathbf{5}, \mathbf{6})}{\delta U(\mathbf{3}, \mathbf{4})} \\
& =-\delta(\mathbf{3}, \mathbf{5}) \delta(\mathbf{4 , 6})-\frac{\delta}{\delta U(\mathbf{3}, \mathbf{4})}\left[v_{\mathrm{H}}(\mathbf{5}) \delta(\mathbf{5}, \mathbf{6})+\Sigma(\mathbf{5}, \mathbf{6})\right] \\
& =-\delta(\mathbf{3}, \mathbf{5}) \delta(\mathbf{4 , 6})-\int d(\mathbf{7 8}) \frac{\delta}{\delta G(\mathbf{7}, \mathbf{8})}\left[v_{\mathrm{H}}(\mathbf{5}) \delta(\mathbf{5}, \mathbf{6})\right. \\
& \quad+\Sigma(\mathbf{5}, \mathbf{6})] \frac{\delta G(\mathbf{7}, \mathbf{8})}{\delta U(\mathbf{3}, \mathbf{4})}
\end{aligned}
$$

where in the last step we used the chain rule (A5). Combining Eqs. (A7) and (A9) we obtain

$$
\begin{aligned}
\frac{\delta G(\mathbf{1}, \mathbf{2})}{\delta U(\mathbf{3}, \mathbf{4})}= & G(\mathbf{1}, \mathbf{3}) G(\mathbf{4}, \mathbf{2}) \\
& +\int d(\mathbf{5 6 7 8}) G(\mathbf{1}, \mathbf{5}) G(\mathbf{6}, \mathbf{2}) \frac{\delta}{\delta G(\mathbf{7 , 8})}\left[v_{\mathrm{H}}(\mathbf{5}) \delta(\mathbf{5}\right. \\
& +\Sigma(\mathbf{5}, \mathbf{6})] \frac{\delta G(\mathbf{7 , 8})}{\delta U(\mathbf{3}, \mathbf{4})}
\end{aligned}
$$

Defining

$$
L_{0}(\mathbf{1}, \mathbf{2}, \mathbf{3}, \mathbf{4})=-i G(\mathbf{1}, \mathbf{3}) G(\mathbf{4}, \mathbf{2}) \text {, }
$$

$$
K(\mathbf{5}, \mathbf{6}, \mathbf{7}, \mathbf{8})=i \frac{\delta}{\delta G(\mathbf{7 , 8})}\left[v_{\mathrm{H}}(\mathbf{5}) \delta(\mathbf{5}, \mathbf{6})+\Sigma(\mathbf{5}, \mathbf{6})\right],
$$

we finally get the Bethe-Salpeter equation

$$
\begin{aligned}
L(\mathbf{1}, \mathbf{2}, 3,4)= & L_{0}(\mathbf{1}, 2,3,4)+\int d(\mathbf{5 6 7 8}) L_{0}(\mathbf{1}, \mathbf{2}, \mathbf{5}, \mathbf{6}) \\
& \times K(\mathbf{5}, \mathbf{6}, 7, \mathbf{8}) L(\mathbf{7}, \mathbf{8}, \mathbf{3}, \mathbf{4}) .
\end{aligned}
$$

Up to this point the derivation has been exact. In order to obtain the working expression for the BSE kernel, $K$, we now make use of the $G W$ approximation to the self-energy. In this case both the Hartree potential $v_{\mathrm{H}}$ and the self-energy $\Sigma$ can be expressed in terms of $G$ :

$$
\begin{gathered}
v_{\mathrm{H}}(\mathbf{1})=\int d(\mathbf{2}) v(\mathbf{1}, \mathbf{2}) \rho(\mathbf{2})=-i \int d(\mathbf{2}) v(\mathbf{1}, \mathbf{2}) G\left(\mathbf{2}, \mathbf{2}^{+}\right), \\
\Sigma(\mathbf{1}, \mathbf{2})=i G(\mathbf{1}, \mathbf{2}) W(\mathbf{1}, \mathbf{2}),
\end{gathered}
$$

which, neglecting the dependence of $W$ on $G$, gives

$$
K(\mathbf{1}, \mathbf{2}, \mathbf{3}, \mathbf{4})=v(\mathbf{1}, \mathbf{3}) \delta(\mathbf{1}, \mathbf{2}) \delta(\mathbf{3}, \mathbf{4})-W(\mathbf{1}, \mathbf{2}) \delta(\mathbf{1}, \mathbf{3}) \delta(\mathbf{2}, \mathbf{4}) .
$$

Here we note that the bare Coulomb interaction is instantaneous $v(\mathbf{1 , 2})=v(1,2) \delta\left(t_{2}-t_{1}\right)$, but this is not in general the case for $W$. Equation (A13) still depends on four times. For our purposes, we want to look at the response at time $t$ from a perturbation at time $t^{\prime}$, that is, our perturbations are local in time. In this case we can express $L$ in terms of the density matrix $\rho(1,2, t)$ as

$$
L(\mathbf{1}, \mathbf{2}, \mathbf{3}, \mathbf{4})=\frac{\delta \rho\left(1,2, t_{1}\right)}{\delta U\left(3,4, t_{3}\right)} \delta\left(t_{1}-t_{2}\right) \delta\left(t_{3}-t_{4}\right),
$$

where we identify $t=t_{1}$ and $t^{\prime}=t_{3}$. Since the initial time is arbitrary for a system in equilibrium - the state of the system does not change in time when we are in the ground state-we furthermore only have to worry about the difference $t^{\prime}-t$. As in the Dyson equation for the Green's function $G$, we can then Fourier transform to get a dependence of only one frequency, thus giving

$$
\begin{aligned}
L(1,2,3,4 \mid \omega)= & L_{0}(1,2,3,4 \mid \omega) \\
& +\int d(5678) L_{0}(1,2,5,6 \mid \omega) \\
& \times K(5,6,7,8 \mid \omega) L(7,8,3,4 \mid \omega) .
\end{aligned}
$$

\section{APPENDIX B: SPIN STRUCTURE OF THE EFFECTIVE BSE AND DEPENDENCE OF THE OCCUPATIONS FOR THE POLARIZABILITY}

\section{Spin structure}

The BSE Hamiltonian can be written in matrix form, as in Eq. (54),

$$
H^{\mathrm{BSE}}=H^{0}+F K
$$

with $K=H^{\text {ex }}+H^{\text {dir }}$ and $F$ given by Eq. (35). We assume a singlet closed shell ground state so the spatial orbitals are the same for spin up and spin down. Explicitly, writing out the spin dependence of the orbitals as $\psi_{i}(1)=\psi_{i}(\boldsymbol{r}) x_{i}(\sigma)$, and $\psi_{i}(2)=\psi_{i}\left(\boldsymbol{r}^{\prime}\right) x_{i}\left(\sigma^{\prime}\right)$, where the spin wave function $x_{i}(\sigma)$ can be either $\alpha(\sigma)$ or $\beta(\sigma)$. Due to orthogonality of the spin wave functions we get

$$
\begin{aligned}
H_{i j, k l}^{0}= & \left(\epsilon_{j}-\epsilon_{i}\right) \delta_{i k} \delta_{j l} \delta_{x_{i} x_{k}} \delta_{x_{j} x_{l}}, \\
H_{i j, k l}^{\mathrm{ex}}= & \int d^{3} r d^{3} r^{\prime} \psi_{i}^{*}(\boldsymbol{r}) \psi_{j}(\boldsymbol{r}) v\left(\boldsymbol{r}, \boldsymbol{r}^{\prime}\right) \psi_{k}\left(\boldsymbol{r}^{\prime}\right) \psi_{l}^{*}\left(\boldsymbol{r}^{\prime}\right) \delta_{x_{i} x_{j}} \delta_{x_{k} x_{l}}, \\
H_{i j, k l}^{\mathrm{dir}}= & -\int d^{3} r d^{3} r^{\prime} \psi_{i}^{*}(\boldsymbol{r}) \psi_{k}(\boldsymbol{r}) W\left(\boldsymbol{r}, \boldsymbol{r}^{\prime}\right) \psi_{j}\left(\boldsymbol{r}^{\prime}\right) \\
& \times \psi_{l}^{*}\left(\boldsymbol{r}^{\prime}\right) \delta_{x_{i} x_{k}} \delta_{x_{j} x_{l}} .
\end{aligned}
$$


This gives the following structure of the problem in the spin indices:

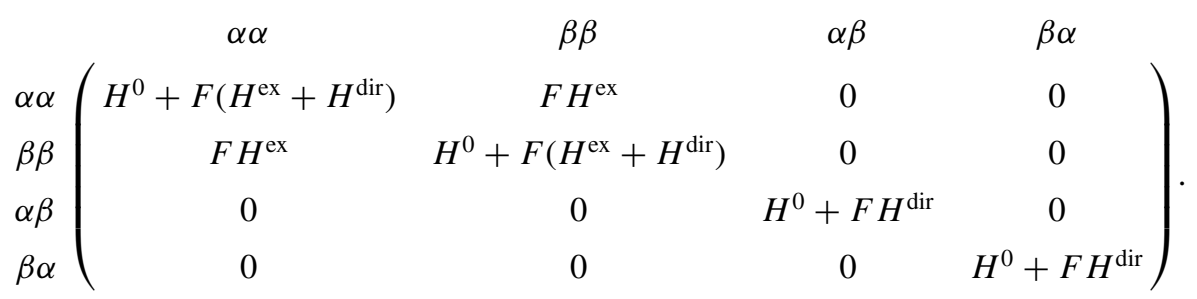

The upper left $2 \times 2$ block can easily be diagonalized to give

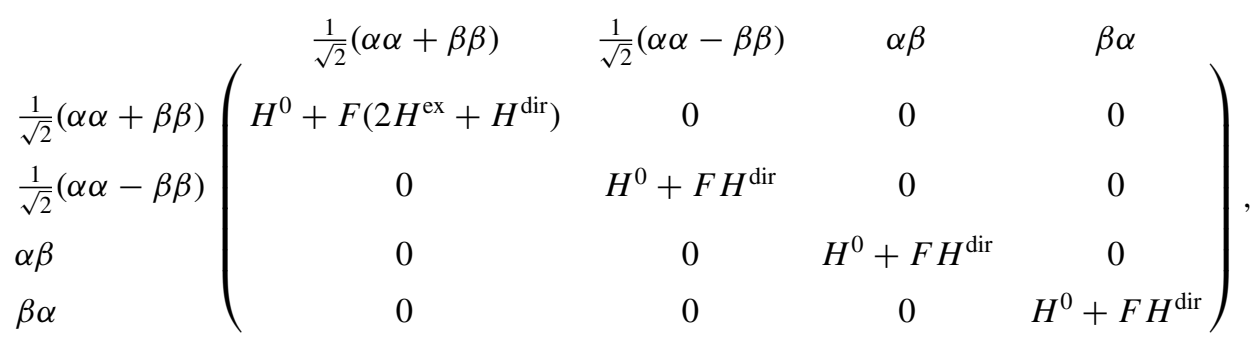

which leads to one singlet solution, where $H^{\mathrm{ex}}$ is included with a factor of 2 , and three triplet solutions where $H^{\mathrm{ex}}$ is absent. Knowing this, we work just with the real-space quantities, remembering to include the correct scaling factor in front of $H^{\mathrm{ex}}$ depending on if we want a singlet or a triplet solution:

$$
K_{i j, k l}^{\text {singlet }}=2 H_{i j, k l}^{\mathrm{ex}}+H_{i j, k l}^{\mathrm{dir}}, \quad K_{i j, k l}^{\mathrm{triplet}}=H_{i j, k l}^{\mathrm{dir}} .
$$

For the dipole elements we have

$$
D_{i j}^{m, \text { singlet }}=\sqrt{2} \int d^{3} r \psi_{i}(\boldsymbol{r})^{*} \boldsymbol{r}_{m} \psi_{j}(\boldsymbol{r}), \quad D_{i j}^{m, \text { triplet }}=0 .
$$

\section{Occupation number structure}

The time-ordered four-point polarizability can be written similarly as in Eq. (21) but with the small imaginary part $i \gamma$ replaced by $i \gamma\left(f_{i^{\prime}}-f_{j^{\prime}}\right)$,

$$
L_{i j, k l}(\omega)=\left\{\left(\omega+i \gamma\left[f_{i^{\prime}}-f_{j^{\prime}}\right)\right] \delta_{i^{\prime} k^{\prime}} \delta_{j^{\prime} l^{\prime}}-H_{i^{\prime} j^{\prime}, k^{\prime} l^{\prime}}^{\mathrm{BSE}}\right\}_{i j, k l}^{-1}\left(f_{k}-f_{l}\right),
$$

which in matrix form is

$$
L(\omega)=\left[(\omega+i \gamma F) I-H^{\mathrm{BSE}}\right]^{-1} F .
$$

From this expression it looks like we have to use all pairs, that is, not only particle-hole and hole-particle pairs but also particle-particle and hole-hole pairs. But actually, only the particle-hole and hole-particle pairs contribute to $L$. To see this we set up the $H^{\mathrm{BSE}}$, and $F$ matrices in blocks corresponding to the $\{v c\},\{c v\},\{v v\}$, and $\{c c\}$ sectors

$$
\begin{aligned}
& H^{\mathrm{BSE}}=\begin{array}{c}
c v \\
v v \\
v v \\
c c
\end{array}\left(\begin{array}{cccc}
v c & c v & v v & c c \\
H^{0}+K & K & K & K \\
-K & H^{0}-K & -K & -K \\
0 & 0 & H^{0} & 0 \\
0 & 0 & 0 & H^{0}
\end{array}\right), \\
& F=\begin{array}{c}
v c \\
v v \\
v v \\
c c
\end{array}\left(\begin{array}{cccc}
I & 0 & 0 & 0 \\
0 & -I & 0 & 0 \\
0 & 0 & 0 & 0 \\
0 & 0 & 0 & 0
\end{array}\right) .
\end{aligned}
$$


This gives the following matrix to be inverted in Eq. (B8):

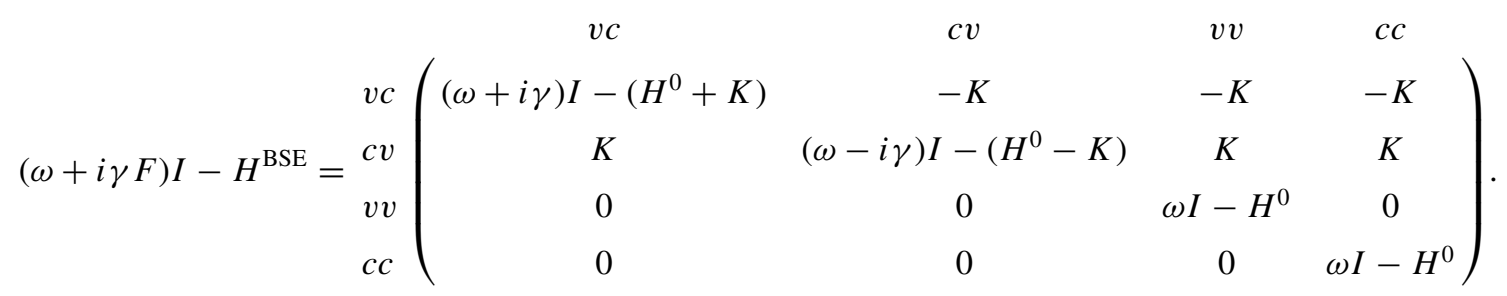

The inverse of a matrix with this block structure is

$$
\left(\begin{array}{cc}
A & B \\
0 & D
\end{array}\right)^{-1}=\left(\begin{array}{cc}
A^{-1} & -A^{-1} B D^{-1} \\
0 & D^{-1}
\end{array}\right)
$$

Looking at the polarizability $L=\left[(\omega+i \gamma F) I-H^{\mathrm{BSE}}\right]^{-1} F$ we see that due to the leftmost $F$ matrix only the $A$ block, that is, the $\{v c\}$ and $\{c v\}$ sectors of $H^{\mathrm{BSE}}$, contribute to $L$. Diagonalizing $H^{\mathrm{BSE}}$ and expanding in left and right eigenvectors gives the following expression:

$$
L_{i j, k l}(\omega)=\sum_{\lambda, \lambda^{\prime}} \frac{A_{i j}^{\lambda} S_{\lambda, \lambda^{\prime}}^{-1} A_{k l}^{\lambda^{\prime} *}\left(f_{k}-f_{l}\right)}{\omega-\epsilon_{\lambda}+\mathrm{i} \gamma\left(f_{i}-f_{j}\right)} .
$$

Note that this is the time-ordered polarizability, the retarded one that we need for the response, that is, Eq. (24), is obtained by setting the sign of the small imaginary part in the denominator to always be positive. We can also use the relations $\operatorname{Im} L^{t}(\omega)=\operatorname{sgn}(\omega) \operatorname{Im} L^{r}(\omega)$ and $\operatorname{Re} L^{t}(\omega)=\operatorname{Re} L^{r}(\omega)$, where the superscript " $t$ " denotes time-ordered and " $r$ " retarded.

[1] E. Runge and E. K. U. Gross, Phys. Rev. Lett. 52, 997 (1984).

[2] M. E. Casida, A. Ipatov, and F. Cordova, Time-Dependent Density Functional Theory (Springer, Heidelberg, 2006).

[3] L. Hedin, Phys. Rev. 139, A796 (1965).

[4] G. Onida, L. Reining, and A. Rubio, Rev. Mod. Phys. 74, 601 (2002).

[5] A. L. Fetter and J. D. Walecka, Quantum Theory of ManyParticle Systems (McGraw-Hill, Boston, 1971).

[6] A. A. Abrikosov, L. P. Gorkov, and I. E. Dzyaloshinski, Methods of Quantum Field Theory in Statistical Physics (Dover, New York, 1963).

[7] W. Hanke and L. J. Sham, Phys. Rev. B 21, 4656 (1980).

[8] F. Bechstedt, K. Tenelsen, B. Adolph, and R. Del Sole, Phys. Rev. Lett. 78, 1528 (1997).

[9] X. Blase and C. Attaccalite, Appl. Phys. Lett. 99, 171909 (2011).

[10] S. Albrecht, G. Onida, and L. Reining, Phys. Rev. B 55, 10278 (1997).

[11] P. Noziéres, Theory of Interacting Fermi Systems (Benjamin, New York, 1964).

[12] L. J. Sham and T. M. Rice, Phys. Rev. 144, 708 (1966).

[13] E. L. Shirley and S. G. Louie, Phys. Rev. Lett. 71, 133 (1993).

[14] G. Onida, L. Reining, R. W. Godby, R. Del Sole, and W. Andreoni, Phys. Rev. Lett. 75, 818 (1995).

[15] L. X. Benedict, E. L. Shirley, and R. B. Bohn, Phys. Rev. Lett. 80, 4514 (1998).

[16] L. X. Benedict, A. Puzder, A. J. Williamson, J. C. Grossman, G. Galli, J. E. Klepeis, J.-Y. Raty, and O. Pankratov, Phys. Rev. B 68, 085310 (2003).

[17] M. Palummo, O. Pulci, R. D. Sole, A. Marini, P. Hahn, W. G. Schmidt, and F. Bechstedt, J. Phys.: Condens. Matter 16, S4313 (2004).

[18] J. M. Garcia-Lastra, J. D. Bass, and K. S. Thygesen, J. Chem. Phys. 135, 121101 (2011).
[19] D. Rocca, D. Lu, and G. Galli, J. Chem. Phys. 133, 164109 (2010).

[20] M. Grüning, A. Marini, and X. Gonze, Comput. Mater. Sci. 50, 2148 (2011).

[21] L. E. Ramos, J. Paier, G. Kresse, and F. Bechstedt, Phys. Rev. B 78, 195423 (2008).

[22] M. Gatti and F. Sottile, Phys. Rev. B 88, 155113 (2013).

[23] L. X. Benedict and E. L. Shirley, Phys. Rev. B 59, 5441 (1999).

[24] A. Sitt, L. Kronik, S. Ismail-Beigi, and J. R. Chelikowsky, Phys. Rev. A 76, 054501 (2007).

[25] M. Rohlfing and S. G. Louie, Phys. Rev. Lett. 80, 3320 (1998).

[26] M. E. Casida, J. Chem. Phys. 122, 054111 (2005).

[27] B. Baumeier, D. Andrienko, and M. Rohlfing, J. Chem. Theory Comput. 8, 2790 (2012)

[28] F. Bruneval and M. A. L. Marques, J. Chem. Theory Comput. 9, 324 (2013).

[29] P. Boulanger, D. Jacquemin, I. Duchemin, and X. Blase, J Chem Theory Comput. 10, 1212 (2014).

[30] C. Ambrosch-Draxl, K. Hummer, S. Sagmeister, and P. Puschnig, Chem. Phys. 325, 3 (2006), Electronic Processes in Organic Solids.

[31] J. Demmel, J. Dongarra, A. Ruhe, and H. van der Vorst, Templates for the Solution of Algebraic Eigenvalue Problems: A Practical Guide, edited by Z. Bai (Society for Industrial and Applied Mathematics, Philadelphia, PA, 2000).

[32] M. Valiev, E. Bylaska, N. Govind, K. Kowalski, T. Straatsma, H. V. Dam, D. Wang, J. Nieplocha, E. Apra, T. Windus, and W. de Jong, Comput. Phys. Commun. 181, 1477 (2010).

[33] Y. Saad, Iterative Methods for Sparse Linear Systems (Society for Industrial and Applied Mathematics, Philadelphia, PA, 2003).

[34] P. Koval, D. Foerster, and O. Coulaud, J. Chem. Theory Comput. 6, 2654 (2010). 
[35] R. Haydock, V. Heine, and M. J. Kelly, J. Phys. C: Solid State Phys. 5, 2845 (1972).

[36] D. Rocca, R. Gebauer, Y. Saad, and S. Baroni, J. Chem. Phys. 128, 154105 (2008)

[37] W. G. Schmidt, S. Glutsch, P. H. Hahn, and F. Bechstedt, Phys. Rev. B 67, 085307 (2003).

[38] C. Attaccalite, M. Grüning, and A. Marini, Phys. Rev. B 84, 245110 (2011).

[39] L. X. Benedict (private communication).

[40] C. Ochsenfeld, J. Kussmann, and D. S. Lambrecht, in LinearScaling Methods in Quantum Chemistry, Reviews in Computational Chemistry (Wiley, New York, 2007), pp. 1-82.

[41] D. Neuhauser, E. Rabani, and R. Baer, J. Phys. Chem. Lett. 4, 1172 (2013).

[42] D. Neuhauser, Y. Gao, C. Arntsen, C. Karshenas, E. Rabani, and R. Baer, Phys. Rev. Lett. 113, 076402 (2014).

[43] E. Rabani, R. Baer, and D. Neuhauser, Phys. Rev. B 91, 235302 (2015).

[44] J. M. Soler, E. Artacho, J. D. Gale, A. García, J. Junquera, P. Ordejón, and D. Sánchez-Portal, J. Phys.: Condens. Matter 14, 2745 (2002).

[45] D. Foerster, P. Koval, and D. Sánchez-Portal, J. Chem. Phys. 135, 074105 (2011)

[46] P. Koval, D. Foerster, and D. Sánchez-Portal, Phys. Rev. B 89, 155417 (2014).

[47] D. Foerster, J. Chem. Phys. 128, 034108 (2008).

[48] W. Kohn and L. J. Sham, Phys. Rev. 140, A1133 (1965).

[49] M. P. Ljungberg, F. Ferrari, P. Koval, and Sánchez-Portal (unpublished).

[50] M. Petersilka, U. J. Gossmann, and E. K. U. Gross, Phys. Rev. Lett. 76, 1212 (1996).

[51] M. J. G. Peach and D. J. Tozer, J. Phys. Chem. A 116, 9783 (2012).

[52] M. J. G. Peach, M. J. Williamson, and D. J. Tozer, J. Chem. Theory Comput. 7, 3578 (2011).

[53] S. Sharifzadeh, P. Darancet, L. Kronik, and J. B. Neaton, J. Phys. Chem. Lett. 4, 2197 (2013).

[54] M. E. Casida, in Recent Advances in Density Functional Methods, Part I, edited by D. P. Chong (World Scientific, Singapore, 1995).
[55] M. Rohlfing and S. G. Louie, Phys. Rev. B 62, 4927 (2000).

[56] X. Ge, S. J. Binnie, D. Rocca, R. Gebauer, and S. Baroni, Comput. Phys. Commun. 185, 2080 (2014).

[57] H. Mori, Prog. Theor. Phys. 34, 399 (1965).

[58] M. H. Lee, Phys. Rev. Lett. 49, 1072 (1982).

[59] Y. Saad, Iterative Methods for Sparse Linear Systems (Society for Industrial and Applied Mathematics, Philadelphia, 2003).

[60] A. Greenbaum, Iterative Methods for Solving Linear Systems (Society for Industrial and Applied Mathematics, Philadelphia, 1997).

[61] Numerical Analysis: Historical Developments in the 20th Century, edited by C. Brezinski and L. Wuytack (Elsevier, Amsterdam, 2001).

[62] A. Mostafazadeh, J. Math. Phys. 43, 205 (2002).

[63] A. Mostafazadeh, J. Math. Phys. 43, 2814 (2002).

[64] R. Bauernschmitt and R. Ahlrichs, J. Chem. Phys. 104, 9047 (1996).

[65] F. Bruneval, J. Chem. Phys. 136, 194107 (2012).

[66] F. Bruneval, S. M. Hamed, and J. B. Neaton, J. Chem. Phys. 142, 244101 (2015).

[67] Experimental data obtained from the NIST Computational Chemistry Comparison and Benchmark DataBase at http://cccbdb.nist.gov, and the NIST webBook at http://webbook.nist.gov.

[68] I. Vasiliev, S. Öğüt, and J. R. Chelikowsky, Phys. Rev. Lett. 82, 1919 (1999).

[69] W. R. Fredrickson and W. W. Watson, Phys. Rev. 30, 429 (1927).

[70] S. P. Sinha, Proc. Phys. Soc, Sect. A 62, 124 (1949).

[71] R. van Harrevelt, J. Chem. Phys. 126, 204313 (2007).

[72] F. Chen and C. Wu, J. Quant. Spectrosc. Radiat. Transfer 85, 195 (2004).

[73] J. C. Grossman, M. Rohlfing, L. Mitas, S. G. Louie, and M. L. Cohen, Phys. Rev. Lett. 86, 472 (2001).

[74] M. L. Tiago and J. R. Chelikowsky, Solid State Commun. 136, 333 (2005).

[75] P. Turchi, F. Ducastelle, and G. Treglia, J. Phys. C: Solid State Phys. 15, 2891 (1982).

[76] F. Bruneval, Ph.D. thesis, Ecole Polytechnique, 2005.

[77] F. Sottile, Ph.D. thesis, Ecole Polytechnique, 2003.

[78] P. C. Martin and J. Schwinger, Phys. Rev. 115, 1342 (1959). 